\title{
Calcul de la pression critique initiale d'une fondation superficielle
}

\section{A. STANCIU}

Faculté de Construction de Iassy

Laboratoire de

Géotechnique Routière et des Ouvrages Souterrains (Roumanie)
On présente un algorithme permettant de déterminer la pression critique initiale dans le sol de fondation sous une semelle superficielle, à partir de la généralisation des théories de Fröhlich-Puzyrevsky, en considérant une extension des domaines plastifiés à la profondeur maximale b/4 (SNIP 2.02.01-83). Sur cette base ont été calculés les coefficients de la pression critique initiale $(\mathrm{M}, \mathrm{M}, \mathrm{M}$ ) pour une charge excentrée et inclinée. On donne également les coefficients correcteurs à appliquer aux formules usuelles pour tenir compte de l'excentricité et de l'inclinaison de la charge.

On montre comment étendre la notion de pression critique initiale aux fondations superficielles isolées, à partir de la pression critique ultime et de la pression admissible.

\section{Calculation of the initial critical pressure under shallow foundations}

The paper presents an algorithmic analysis of the initial critical pressure on the foundation soil for a continuous strip footing by generalizing the Fröhlich-Puzyrevsky theory and assuming an extent of the plastic zones having a maximum depth, equal to b/4 (SNIP 2.02.01-83). On this basis the coeficients of the initial critical pressure $\left(\mathrm{M}_{r}, \mathrm{M}_{0}, \mathrm{M}\right)$ ) are calculated for an inclined eccentric force. The correction factors to be applied to usual formulas for taking into account eccentricity and inclination of the load, are calculated as well.

It is shown how to extend the concept of the initial critical pressure to spread footings on the basis of the ultimate critical pressure and of the allowable pressure. 


\section{Généralités}

La détermination des caractéristiques géométriques d'une fondation superficielle (longueur, largeur, profondeur) doit être réalisée de telle sorte que les tassements estimés (s) soient inférieurs aux tassements admissibles $\left(\mathrm{s}_{\mathrm{ad}}\right)$ tout en évitant toute perte de stabilité, ce qui est fait en limitant la pression $\left(\mathrm{q}_{\max }\right.$ ) sous la base de la fondation à des valeurs inférieures à une valeur imposée $\left(\mathrm{q}_{\mathrm{ad}}\right.$ - admissible/ $\mathrm{R}$ - de calcul).

Les méthodes de calcul pour estimer les tassements (s) reposent sur l'équation de Boussinesq qui permet d'obtenir les contraintes et les déformations dans le terrain de fondation en considérant que le comportement de ce dernier est élastique, homogène et isotrope.

Mais le terrain ne possède, à vrai dire, aucune de ces caractéristiques, ce qui peut être mis en évidence en comparant la courbe réelle contrainte-déformation d'un terrain d'une part et celle d'un milieu élastique d'autre part (Schlosser, 1983), ainsi qu'en analysant la forme de la courbe pression moyenne - tassement résultant du chargement par paliers d'une fondation superficielle (Tsytovich, 1976; Krol, 1971).

Cependant, lors du tassement d'une fondation on peut distinguer deux phases dans les déformations du terrain (Tsytovich, 1976 ; Costet et Sanglerat, 1969), (Fig. 1): a) une phase de compression (Fig. 1c) dans laquelle le tassement $\left(\mathrm{s}_{\mathrm{c}}\right)$ correspond à de la consolidation primaire et résulte d'une réduction de l'indice des vides ;

b) une phase de cisaillement (Fig. 1c-d), caractérisée par des déformations angulaires qui se matérialisent par des tassements plastiques ( $\mathrm{s}_{\mathrm{p}}$ ).

Au cours de la première phase, la relation entre déformation et pression peut être considérée comme approximativement linéaire (Tomlinson, 1969).

Cela permet d'appliquer les relations résultant des équations de Boussinesq (Schlosser, 1983), tant au calcul de l'état des contraintes qu'à celui des déformations du terrain de fondation. La charge qui correspond à la fin de la phase de compression et qui limite le domaine de comportement linéaire du terrain avant la phase de cisaillement (Krol, 1971: Tsytovich, 1976) est définie comme la charge critique initiale (in. $Q_{v T}$ ), la pression correspondante étant définie comme la pression critique initiale (in. $p_{c r}$ ).

Pour les charges $Q_{1}>$ in. $Q_{c}$ ce sont les déformations plastiques qui prédominent, la relation pression-tassement devenant non linéaire et les tassements plastiques observés ne pouvant plus être calculés à partir de modèles reposant sur l'équation de Boussinesq. La charge maximale, qui limite cette phase et qui correspond au développement complet du cône de compression et des lignes de glissement, est définie comme

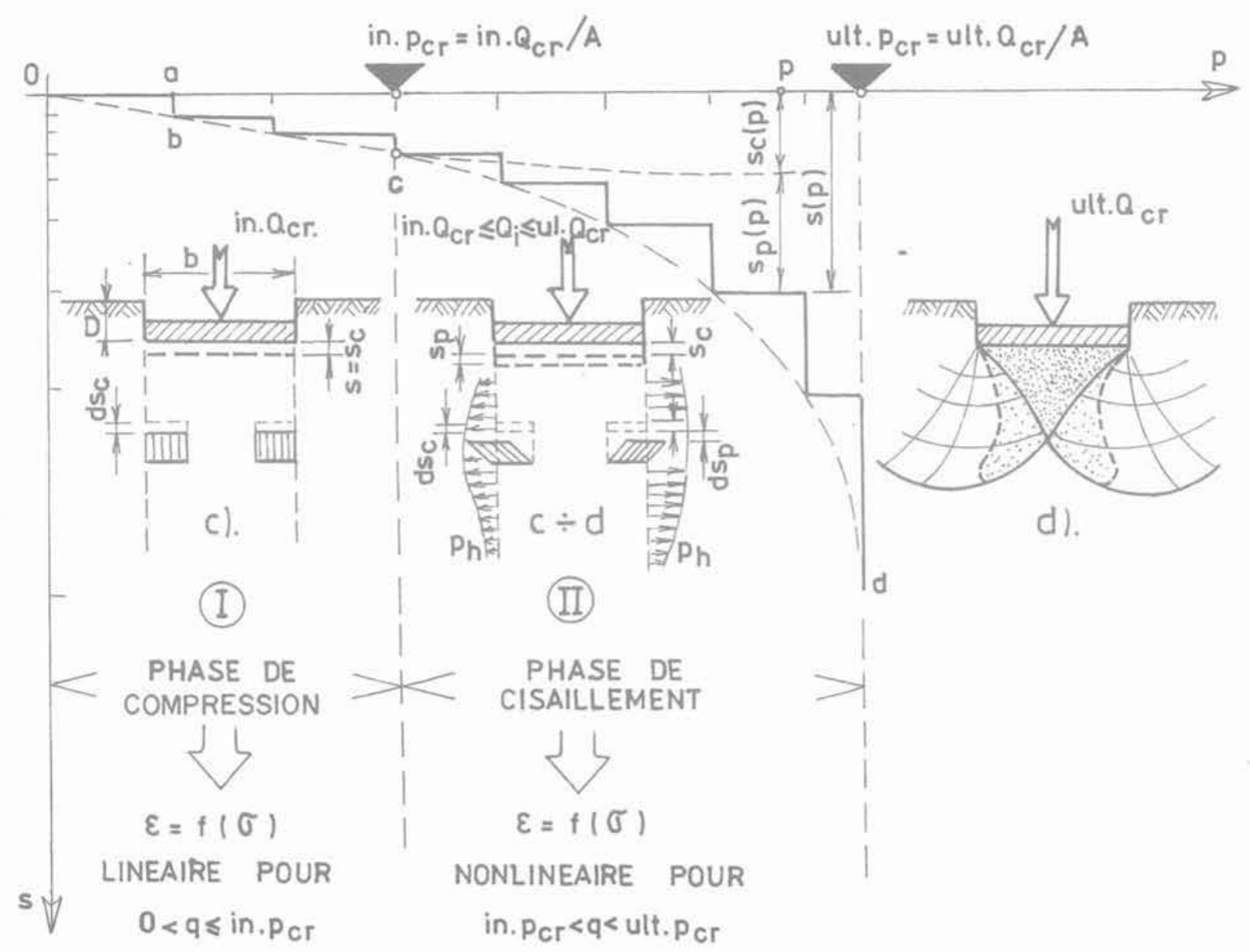

FIG.1 Relation entre tassement de la fondation et pression moyenne au cours du chargement : a - charges appliquées par paliers; $\mathbf{b}$ - partie initiale de la courbe de tassement; $\mathbf{c}$ - fin de la phase de compression et début du cisaillement; $\mathrm{d}$-lignes de glissement et cône de compression à l'équilibre limite. 
étant la charge critique limite (ult. $Q_{c}$ ), et la pression correspondante, la pression critique ultime $\left(\mathrm{q}_{\mathrm{u}} /\right.$ ult. $\left.\mathrm{p}_{\mathrm{cr}}\right)$.

La définition de la pression admissible $\left(\mathrm{q}_{\mathrm{ad}}\right)$ pour une fondation donnée (Schlosser, 1983) requiert que soient vérifiées les deux inégalités:

$\mathrm{q}_{\mathrm{ad}} \leq$ in. $\mathrm{p}_{\mathrm{cr}}$ : pour pouvoir calculer les efforts et les déformations dans le sol en assimilant celui-ci à un milieu linéairement déformable:

$\mathrm{q}_{\mathrm{ad}} \leq$ ult.p $\mathrm{p}_{\mathrm{c}} / \mathrm{F}_{\mathrm{s}}$ : pour éviter toute rupture du sol.

Dans le cas où l'on accepte que la pression admissible soit supérieure à la pression critique initiale $\left(\mathrm{q}_{\mathrm{ad}}\right\rangle$ in. $\mathrm{p}_{\mathrm{cr}}$ ) tout en respectant la condition $\mathrm{q}_{\mathrm{nd}} \leq$ ult. $\mathrm{p} / \mathrm{F}$, le tassement total de la fondation sera déterminé par (Krol, 1971);

$$
\mathrm{s}=\mathrm{s}_{\mathrm{c}}\left(\mathrm{in} \cdot \mathrm{p}_{\mathrm{cr}}\right)+\mathrm{s}_{\mathrm{p}}\left(\Delta \mathrm{p}=\mathrm{q}_{\mathrm{ad}}-\text { in. } \mathrm{p}_{c r}\right)
$$

où:

s est le tassement calculé à l'aide des méthodes classiques qui considèrent le sol comme un milieu linéairement déformable (Bowles, 1979) ;

S. est le tassement plastique calculé de façon approchée par la méthode de Fröhlich-Koegler - Scheiding (Krol, 1971).

\section{2}

\section{Détermination \\ de la pression critique initiale pour une semelle continue}

L'expression de la pression critique initiale, définie précédemment comme la pression correspondant à la fin de la phase de compression et au début de l'apparition des zones de cisaillement ou zones plastiques, a été établie (selon Tsytovich, 1976) par Puzyrevsky en 1929, Ghersevanov en 1930 et Fröhlich en 1934. Ainsi, en associant l'état de contraintes dans le semi-plan linéairement déformable, sous l'effet d'efforts régulièrement distribués (Fig. 2), avec le critère de rupture des sols de Mohr-Coulomb (Verdeyen et al., 1971; Tsytovich, 1976) qui s'écrit:

$$
\sin \varphi=\left(\sigma_{1}-\sigma_{2}\right) /\left(\sigma_{1}+\sigma_{2}+2 \operatorname{cotg} \varphi\right)
$$

on obtient :

$$
p_{c r}=\frac{\pi}{\operatorname{ctg} \varphi+\varphi-\frac{\pi}{2}}\left(\gamma z_{\text {max }}+\gamma D+c \operatorname{cotg} \varphi\right)+\gamma D(2)
$$

où:

$\mathrm{z}_{\max }$ représente la profondeur maximale d'extension des zones plastiques.

Puis en imposant la condition (Puzyrevscky, 1929; Fröhlich, 1934) qu'en aucun point, sous la base de la fondation, la contrainte tangentielle ne doit dépasser la résistance au cisaillement du sol $\left(\mathrm{z}_{\max }=0\right.$, soit pas de déformation plastique), on obtient lexpression de la pression critique initiale, qui peut être mise sous la forme:

$$
\text { in. } \left.p_{c T}=\gamma D M_{q}+c M_{c}\right)
$$

où:

$q=\gamma D$ : surcharge au niveau de la base de la fondation : $\mathrm{c}=$ cohésion du sol $\varphi=$ angle de frottement interne du sol

avec:

$$
\begin{gathered}
M_{\mathrm{q}}=1+\pi /(\operatorname{cotg} \varphi+\varphi-\pi / 2) \\
\mathrm{M}_{c}=\pi .(\operatorname{cotg} \varphi+\varphi-\pi / 2)
\end{gathered}
$$

La connaissance de la pression critique initiale, déterminée à partir de $z_{\max }=0$ et donnée par la relation (3a), permet d'assurer la sécurité des structures (Tsytovich, 1976). Il n'est plus nécessaire d'introduire un coefficient de sécurité, considérant alors que $\mathrm{q}_{\mathrm{ad}}=$ in. $\mathrm{p}_{\mathrm{cr}}$

Trouvant la condition $z_{\text {mix }}=0$ très restrictive et aussi pour introduire l'effet de la largeur de la fondation dans le calcul de la pression critique initiale, N.N. Maslov (1982) a imposé la valeur $z_{\max }=\mathrm{b} \operatorname{tg} \varphi$. Quant aux normes soviétiques (SNIP II - B.1.62/SNIP II - 1574/SNIP 2.02.01-83), ainsi que les normes roumaines (STAS 3300/2-85), elles considèrent une extension maximale en profondeur des zones plastiques égale à b/4 (Tsytovich, 1976).

$$
\begin{aligned}
& \sigma_{1}=\frac{p-\gamma \cdot D}{\pi}(\alpha+\sin \alpha)+\gamma(D+Z) \\
& \sigma_{2}=\frac{p-\gamma}{\pi}(\alpha-\sin \alpha)+\gamma(D+Z)
\end{aligned}
$$

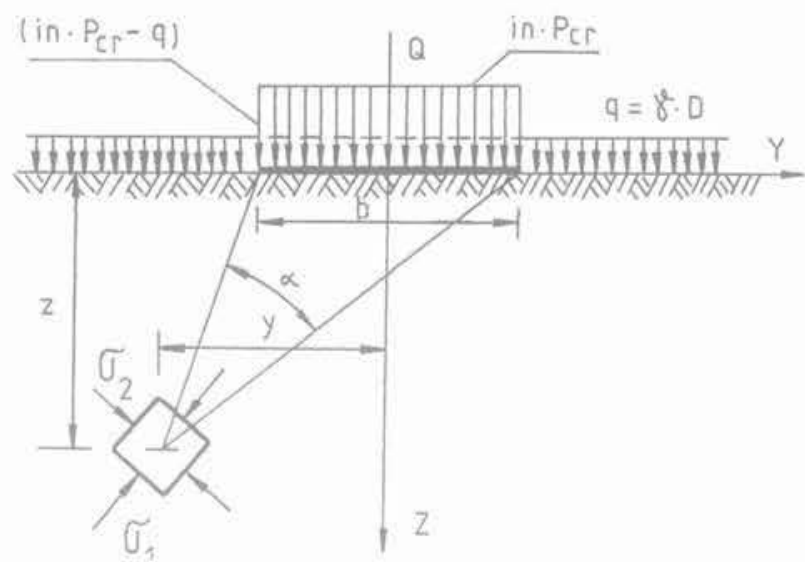

FIG. 2 Schéma de calcul de la théorie de Puzyrevsky-Frölich.

En retenant la valeur $z_{m a}=b / 4$, recommandée par les normes SNIP II-B.1-62 et utilise dans les actuelles normes soviétiques (SNIP 2.02.01-83), l'expression de la pression critique initiale peut être mise sous la forme:

$$
\text { in. } p_{c r}=\gamma \cdot b \cdot M+q M_{q}+c M_{c}
$$

où : $M=0,25 \cdot \pi /(\operatorname{cotg} \varnothing+\varnothing-\pi / 2)$

et où: $M_{q}$ et $M_{c}$ sont les expressions données en ( $3 b$ ).

Pour les charges excentrées et inclinées sur les fondations, il est nécessaire de calculer les valeurs des coefficients $M_{*} ; M_{0} ; M_{c}$ ) en fonction de l'inclinaison relative $(\delta / \varphi)$ et de l'excentricité relative $(e r=e / b)$ de la force appliquée (fig. 3).

Le calcul de ces valeurs, à partir de solutions analytiques et selon la méthode de Puzyrevsky-Fröhlich, n'est pas possible, compte tenu des complications mathématiques qui en résultent. Le problème peut être résolu numériquement en supposant une répartition linéaire de la contrainte sous la base de la fondation, 

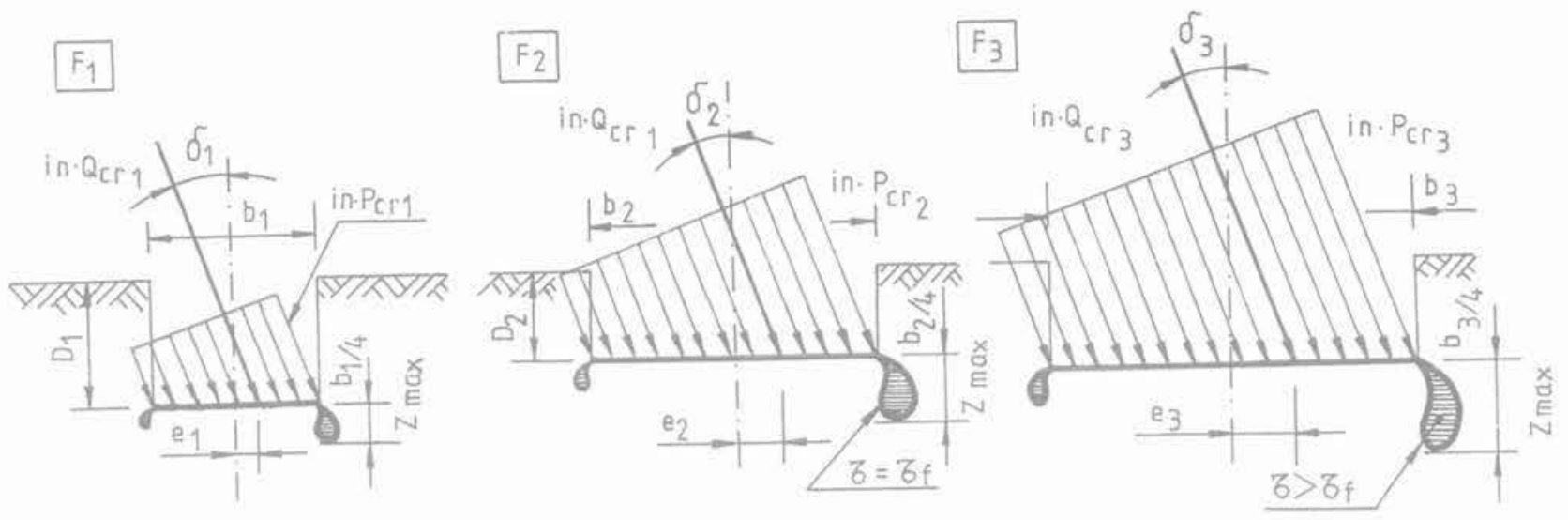

$$
\begin{aligned}
& b_{1} \neq b_{2} \neq b_{3} ; D_{1} \neq D_{2} \neq D_{3} ; \gamma_{1} \neq \gamma_{2} \neq \gamma_{3} ; c_{1} \neq c_{2} \neq c_{3} \\
& \phi_{1}=\phi_{2}=\phi_{3} ; \delta_{1}=\delta_{2}=\delta_{3} ; e_{1} / b_{1}=e_{2} / b_{2}=e_{3} / b_{3}
\end{aligned}
$$

FG.3 Schéma de calcul et paramètres dans la théorie de Puzyrevsky-Fröhlich généralisée.

sous l'action de la force in. $\mathrm{Q}_{\mathrm{cri}}$, et en considérant que la valeur maximale de cette contrainte correspond à la pression critique initiale (Fig. 3). Pour cela, on suppose que la pression critique initiale, donnée par une relation de type (4), est connue et que les valeurs de ses coefficients $\left(M_{y} ; M_{q} ; M_{c}\right)$ sont des inconnues. La relation (4) peut ainsi être considérée comme une équation à trois inconnues $\left(\mathrm{M}_{\gamma} ; \mathrm{M}_{\mathrm{c}} ; \mathrm{M}_{\mathrm{c}}\right)$, et en $\mathrm{y}$ association trois fondations quelconques $\left(\mathrm{F}_{1} ; \mathrm{F}_{2} ; \mathrm{F}_{3}\right)$, de dimensions différentes, mais placées dans des terrains de fondation ayant le même angle de frottement interne, et des forces critiques initiales (in. $Q_{\text {cri }}$ ) ayant la même inclinaison $(\delta / \varnothing)$, mais des excentricités relatives $\left(e r=e_{j} / b_{j}\right)$ différentes, il résulte que les expressions des pressions critiques initiales (correspondant à ces trois fondations quelconques) forment un système de trois équations à trois inconnues admettant une solution unique, soit:

$$
\left\{\begin{array}{l}
\gamma_{1} b_{1} M_{r}+q_{1} M_{q}+c_{1} M_{c}=\text { in. } p_{c r 1} \\
\gamma_{2} b_{2} M_{r}+q_{2} M_{q}+c_{2} M_{c}=\text { in. } p_{c r 2} \\
\gamma_{3} b_{3} M_{\gamma}+q_{3} M_{q}+c_{3} M_{c}=\text { in. } p_{c r 3}
\end{array}\right.
$$

La solution du système (5) est:

$$
M_{\gamma}=\Delta_{1} / \Delta M_{\theta}=\Delta_{2} / \Delta M_{c}=\Delta_{3} / \Delta
$$

où :

$$
\begin{aligned}
& \Delta=\gamma_{1} b_{1} A_{1}-q_{1} B_{1}+c_{1} C_{1} \\
& \Delta_{1}=\operatorname{in~} p_{\text {er. } 1} A_{1}-q_{1} B_{2}+c_{1} C_{2} \\
& \Delta_{2}=\gamma_{1} b_{1} B_{2}-\text { in. } p_{c r 1} B_{1}+c_{3} C_{3} \\
& \Delta_{3}=-\gamma_{1} b_{1} C_{2}-q_{1} C_{3}+\text { in. } p_{c \cdot 1} C_{1}
\end{aligned}
$$

et:

$$
\begin{aligned}
& A_{1}=q_{2} c_{3}-c_{2} q_{3} \\
& B_{1}=\gamma_{2} b_{2} c_{3}-\gamma_{3} b_{3} c_{2} \\
& C_{1}=\gamma_{2} b_{2} q_{3}-\gamma_{3} b_{3} q_{2} \\
& B_{2}=\text { in. } p_{c r \cdot 2} c_{3}-\text { in. } p_{c r 3} c_{2} \\
& C_{2}=\text { in. } p_{c r \cdot 2} q_{3}-\text { in. } p_{c \cdot 3} q_{2} \\
& C_{3}=\gamma_{2} b_{2} \text { in. } p_{c r \cdot 3}-\gamma_{3} b_{3} \text { in. } p_{c r .2}
\end{aligned}
$$

Dans les expressions (6) les valeurs des pressions critiques initiales (in. $\mathrm{p}_{\mathrm{cr}}$ ) correspondant aux trois fondations quelconques $(i=1,2,3)$ sont des inconnues implicites.
Leur détermination, dans le cas d'une répartition linéaire de la contrainte sous la base est faite à l'aide de la relation:

$$
\text { in. } \mathrm{p}_{\mathrm{cr} i \mathrm{i}}=\mathrm{in} \cdot \mathrm{Q}_{\mathrm{cr} i}(1+6 \mathrm{er}) / \mathrm{b}_{\mathrm{i}}
$$

où :

er $=e_{1} / b_{1}$ : excentricité relative de la force ;

in. $Q_{c, i}$ : force critique initiale d'inclinaison $(\delta)$, d'excentricité relative (er) conduisant à une profondeur de la zone plastique sous la semelle égale à $\mathrm{b}_{1} / 4$.

La force critique initiale, pour chacune des trois fondations, peut être déterminée par un processus itératif d'interpolation linéaire (Fig. 4), jusqu'à ce que l'on obtienne:

$$
z_{\max }=b_{1} / 4 \pm \varepsilon
$$

à l'aide de la relation:

$$
\begin{gathered}
\text { in. } Q_{c r i}=Q_{2}+\left[\left(Q_{1 i}-Q_{2 i}\right)\left(0,25 b_{i}-z_{\max 22}\right)\right] y \\
/\left(z_{\max 11}-z_{\max 2}\right)
\end{gathered}
$$

où $Q_{1 j}$ et $Q_{2 i}$ sont deux forces d'intensité quelconques, prises comme valeurs initiales et respectant les conditions:

$$
\mathrm{Q}_{21}<\text { in. } \mathrm{Q}_{\mathrm{ct} 1}<\mathrm{Q}_{11} \text { et } \mathrm{z}_{\max 21}<\mathrm{b} / 4<\mathrm{z}_{\max 1 .}
$$

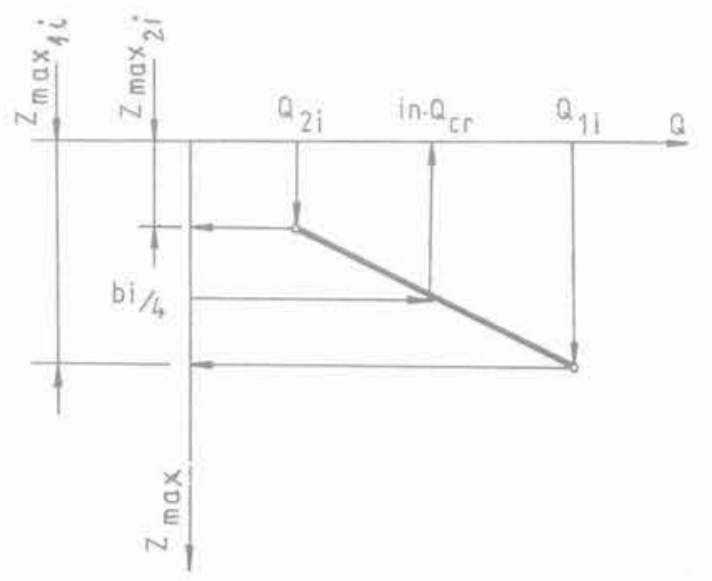

FIG.4 Détermination de la force critique initiale par interpolation linéaire. 


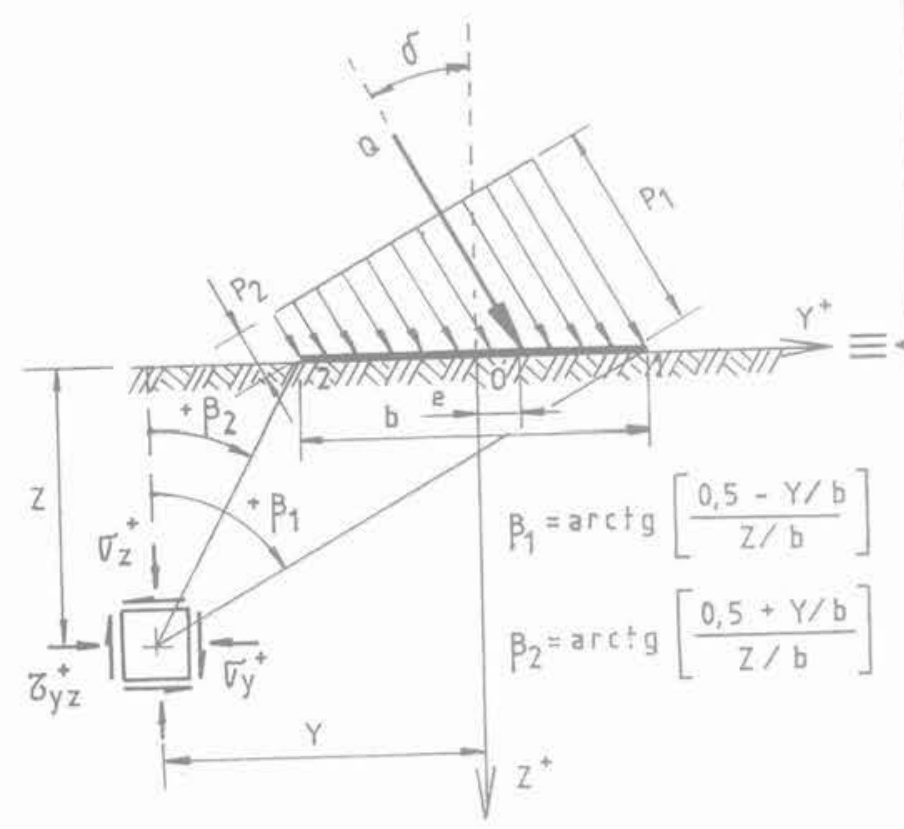

$P_{1,2}=Q \cdot[1 \pm 6 \cdot e r] / b$
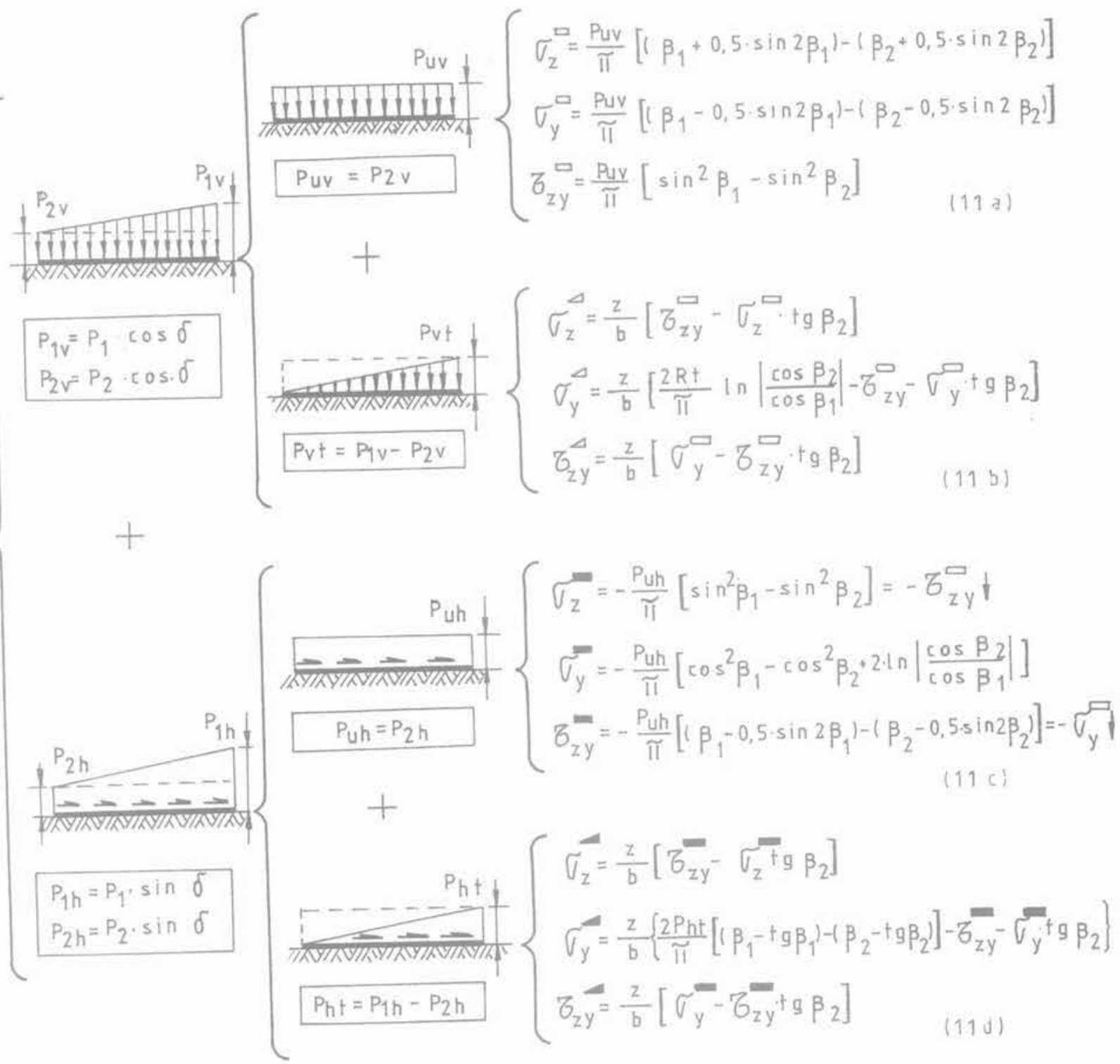
La détermination de la profondeur maximum de dévelopement de la zone plastique $z_{\max 11}\left(\right.$ et $z_{\max 2}$ ) sous l'action d'une force donnée $Q_{1}\left(\right.$ et $\left.Q_{2}\right)$ se fait en cherchant la valeur maximale de la profondeur (z) pour laquelle la fonction:

$$
f\left(y, z, p_{1}, p_{2}\right)=\left[\left(\theta_{\max }-\varnothing\right) \pm \varepsilon\right]
$$

a une valeur positive ou nulle.

$y \in[0,01 ; 1,5 b]$ et $z \in[0,01 ; 1,5 b]$ sont les coordonnées d'un point courant du demi-plan:

$p_{1} ; p_{2}$ : les valeurs extrêmes du diagramme de répartition des contraintes sous la base (Fig. 5);

$\theta_{\max } / \varphi$ : le rapport de l'angle de déviation maximum à l'angle de frottement interne du sol

$\varepsilon$ : le degré de précision.

La condition $\left[\left(\theta_{\max }-\varphi \pm \varepsilon\right] \geq 0\right.$ indique que le critère de plasticité de Mohr-Coulomb est violé. L'algorithme de la recherche de $z_{\max }$ comprend une variante de la méthode du nombre d'or (Press et al., 1986). L'angle de déviation maximum est donné par la relation suivante (Tsytovich, 1976):

$$
\theta_{\operatorname{mix}}=\arcsin \left[\frac{\sqrt{\left(\sigma_{z}-\sigma_{y}\right)^{2}+4 \tau_{z y}^{2}}}{\left(\sigma_{z}+\sigma_{y}\right)+2 \operatorname{ctg} \varnothing}\right]
$$

où :

$\sigma_{z}=\Sigma \sigma_{p z}+\gamma(z+D)$

$\sigma_{y}=\Sigma \sigma_{p y}+K_{0} \cdot \gamma(\mathrm{z}+\mathrm{D})$

$\tau_{z y}=\Sigma \tau_{z y}$

et où :

$\Sigma \sigma_{p z^{\prime}}, \Sigma \sigma_{p y^{\prime}}, \Sigma \tau_{z y}$ sont les contraintes provoquées par la force et résultant de l'addition des contraintes calculées à partir des relations (11) (Stanciu, Boti, 1986); $\mathrm{K}_{0}$ est le coefficient de poussée dans l'état de repos.

Dans les relations (11b) à (11d), les quantités $\sigma_{z^{\prime}} \sigma_{y^{\prime}}$ $\sigma_{z y^{\prime}} \sigma_{z^{\prime}} \sigma_{y}, \sigma_{z y}$ sont remplacées par les expressions correspondantes données par les relations (11a) à (11c) où l'on introduit les contraintes $p_{\mathrm{yt}}$ et $\mathrm{p}_{\mathrm{ht}}$ à la place des contraintes $p_{u v}$ et $p_{u h}$ (Fig. 5).

Conformément à ce qui vient d'être présenté ci-dessus, un logiciel, appelé Prelim, a été développé en langage Fortran et en langage Pascal. Les données d'entrée (Fig. 3) en sont :

- les dimensions des trois fondations $\left(F_{1}, F_{2}, F_{3}\right)$ arbitrairement choisies et les caractéristiques du terrain d'appui:

$\mathrm{b}_{1}=1,30 \mathrm{~m} \quad \mathrm{D}_{1}=2,00 \mathrm{~m} \quad \gamma_{1}=18,70 \mathrm{KN} / \mathrm{m}^{3} \quad \mathrm{c}_{1}=21,00 \mathrm{KPa}$

$\mathrm{b}_{2}=3,50 \mathrm{~m} \quad \mathrm{D}_{2}=1,50 \mathrm{~m} \quad \gamma_{2}=19,00 \mathrm{KN} / \mathrm{m}^{3} \quad \mathrm{c}_{2}=22,00 \mathrm{KPa}$

$\mathrm{b}_{3}=6,00 \mathrm{~m} \quad \mathrm{D}_{3}=2,25 \mathrm{~m} \quad \gamma_{3}=15,80 \mathrm{KN} / \mathrm{m}^{3} \quad \mathrm{c}_{3}=16,00 \mathrm{KPa}$

- les caractéristiques des forces appliquées sur ces fondations:

$$
\delta_{i} / \varphi \mathrm{i}=0,00 ; 0,25 ; 0,50 ; 0,75 ; 1,00
$$

$\mathrm{er}_{\mathrm{i}}=0,0 ; 0,046666667 ; 0,08333333 ; 0,125 ; 0,16666667$

- l'angle de frottement interne (le même pour toutes les trois fondations) :

$$
\varphi \mathrm{i}=1^{\circ}, 5^{\circ}, 10^{\circ}, 15^{\circ}, 20^{\circ}, 25^{\circ}, 30^{\circ}, 35^{\circ}, 40^{\circ} \text {. }
$$

Le numéro de conditionnement de la matrice du système (5) sera de l'ordre de $10^{2}$, ce qui implique un degré de précision de $10^{-7}$ à $10^{-8}$ pour le calcul des élé- ments de la matrice, afin que l'erreur sur le résultat ne dépasse pas $10^{-4}$ à $10^{-5}$.

Le logiciel permet d'obtenir les grandeurs suivantes: - les forces critiques initiales in. $\mathrm{Q}_{\mathrm{cri} .}$ et les profondeurs maximales des zones plastiques $z_{\max i}=b \pm \varepsilon(\varepsilon=1 \mathrm{E}-7)$ correspondant aux trois fondations, pour les valeurs données des paramètres $\varnothing,(\delta / \varnothing)$ et $(e r)$;

- les contraintes critiques initiales (in. $\mathrm{p}_{\mathrm{cr.1}}=\mathrm{p}_{\mathrm{li}}$ ) pour les trois fondations;

- les coefficients $\left(\mathrm{M}_{\gamma}, \mathrm{M}_{\mathrm{q}^{\prime}} \mathrm{M}_{\mathrm{e}}\right)$ de la contrainte critique initiale.

Les valeurs des coefficients de la contrainte critique initiale, calculées en fonction de l'angle de frottement interne $(\varnothing)$, de l'excentricité relative (er) et de l'inclinaison de la force $(\delta / \varnothing)$, pour $K_{0}=1$, sont présentées dans l'annexe 1.

Ces valeurs peuvent être utilisées pour le calcul de la pression critique initiale (à partir de la relation 4). En outre, on peut calculer la force critique initiale (in. $Q$ ), pour une excentricité et une inclination données, par la relation suivante:

$$
\text { in. } Q_{c r}=\text { in. } p_{c r} b /(1+6 e / b)
$$

Les valeurs des forces critiques initiales pour $\varnothing=$ $25^{\circ}$, correspondant aux trois fondations $\left(F_{1}, F_{2,}, F_{3}\right)$, sont données dans l'annexe $2 \mathrm{a}$. L'annexe $2 \mathrm{~b}$ donne les valeurs des forces critiques initiales pour $\delta \neq 0$ et er $\neq 0$ en pourcentage des forces critiques initiales correspondant à $\delta_{1}=0$ et er $=0$.

L'annexe $2 c$ donne les valeurs des forces critiques initiales (pour $\delta \neq 0$ et er $\neq 0$ ) en pourcentage des valeurs des forces critiques initiales correspondant à $\delta$ $=0$ et $\operatorname{er} \neq 0$.

L'annexe 3 présente de façon analogue les valeurs des tables donnant les valeurs des forces et pressions critiques initiales, ainsi que des coefficients $M_{\gamma} ; M_{n} ; M_{\text {f }}$ en fonction des différentes valeurs déjà mentionnées de l'angle de frottement interne du sol.

Les tables 3 a donnent les valeurs des forces critiques, des pressions critiques initiales et des coefficients $\mathrm{M}$, rapportées aux valeurs des mêmes grandeurs pour la charge critique verticale centrée.

En examinant ces tables on constate que:

- les rapports des forces critiques initiales, des pressions critiques initiales et des coefficients de $M$ (pour $\delta$ $=0$ ) aux valeurs des mêmes grandeurs correspondant à la charge critique verticale centrée sont pratiquement indépendantes de l'angle de frottement interne;

- les valeurs des forces critiques initiales diminuent quand l'excentricité augmente et celles de la pression critique, ainsi que celles des coefficients $\mathrm{M}$ augmentent avec l'excentricité :

- les rapports des coefficients $\left(\mathrm{M}_{\gamma} ; \mathrm{M}_{q} ; \mathrm{M}_{\mathrm{c}}\right)$ ont pratiquement des valeurs identiques aux rapports de la pression critique initiale.

Prenant pour base les valeurs moyennes de ces rapports, on a défini les coefficients de corrélation suivants qui dépendent de l'excentricité relative:

- le coefficient de corrélation de la force critique initiale:

$$
e_{\mathrm{F}}=\text { in. } Q_{c r}(\delta=0 ; e r \neq 0) / \text { in. } Q_{c r}(\delta=0 ; \text { er } \neq 0)
$$

- le coefficient de corrélation de la pression critique initiale et de ses coefficients:

$$
e_{p}=\text { in. } p_{c r}(\delta=0 ; e r \neq 0) / i n \cdot p_{c r}(\delta=0 \text {; er }=0)
$$

En utilisant la méthode des moindres carrés (Dorn, McCracken, 1972) on montre que les coefficients de 
corrélation ont des expressions du troisième degré en fonction de l'excentricité relative des charges qui s'exercent sur les fondations:

$$
\begin{gathered}
e_{F}=1,00+2,76 e r-12,02(e r)^{2}+21,21(e r)^{3} \\
e_{p}=1,00-3,22 e r+6,21(e r)^{2}-5,67(e r)^{3}
\end{gathered}
$$

Les graphiques correspondant aux équations (15) et (16) sont présentés à la figure 6 .

En procédant de la même manière sur les moyennes des rapports des forces critiques initiales, des pressions critiques initiales et des coefficients $M$ (présentées dans des tables du type de l'annexe $2 c)$, on a obtenu (annexe 3 ) l'influence de l'inclinaison $(\delta)$ de la charge sur les trois grandeurs (in. $Q_{c r}$; in. $p_{c r} ; M_{1}$ ). De l'analyse des valeurs des rapports présentés dans l'annexe $3 \mathrm{~b}$ en fonction de l'angle de frottement interne et de l'inclinaison de la charge, il résulte les points suivants:

- les rapports moyens (pour $\delta=$ cte; annexe $2 c$ ), obtenus en divisant par les valeurs correspondant à la sollicitation verticale excentrée $(\delta=0$ et er $€$ [0; 0,1666666]), sont pratiquement les mêmes pour les forces critiques initiales, les pressions critiques initiales et les coefficients $M_{\gamma} ; M_{q} ; M_{c}$;

- les coefficients de corrélation $i_{y} ; i_{0} ; i_{f}$ qui rendent compte de l'effet de l'inclinaison de la charge (similaires aux coefficients sur l'inclinaison dans l'expression de la capacité portante du sol de fondation) sont tous égaux, $\mathrm{i}=\mathrm{i}_{\gamma}=\mathrm{i}_{q}=\mathrm{i}_{\mathrm{c}}$, et varient en fonction de $\varnothing,(\delta / \varnothing)$ et er; - l'erreur maximale faite en prenant les valeurs moyennes, pour $\delta=$ constant et er $\neq 0$ (annexe 2c), est d'environ $\pm 6 \%$ sur l'inclinaison maximum $\delta / \varnothing=1,00$ et sur l'excentricité maximum er =0,16666667.

Les variations du facteur d'inclinaison (i) en fonction de l'angle de frottement interne et de l'inclinaison relative de la charge sont présentées à la figure 7. Pour éviter tout glissement de la fondation sur sa base, l'inclinaison doit respecter les conditions suivantes (SNIP 2.02.01-82; Bowles, 1982):

$$
\begin{gathered}
\operatorname{tg} \delta \leq \sin \varphi_{\mathrm{I}} \\
\mathrm{H} \leq \mathrm{V} \operatorname{tg} \delta^{\prime}+\mathrm{c}_{\mathrm{a}} \mathrm{A}
\end{gathered}
$$

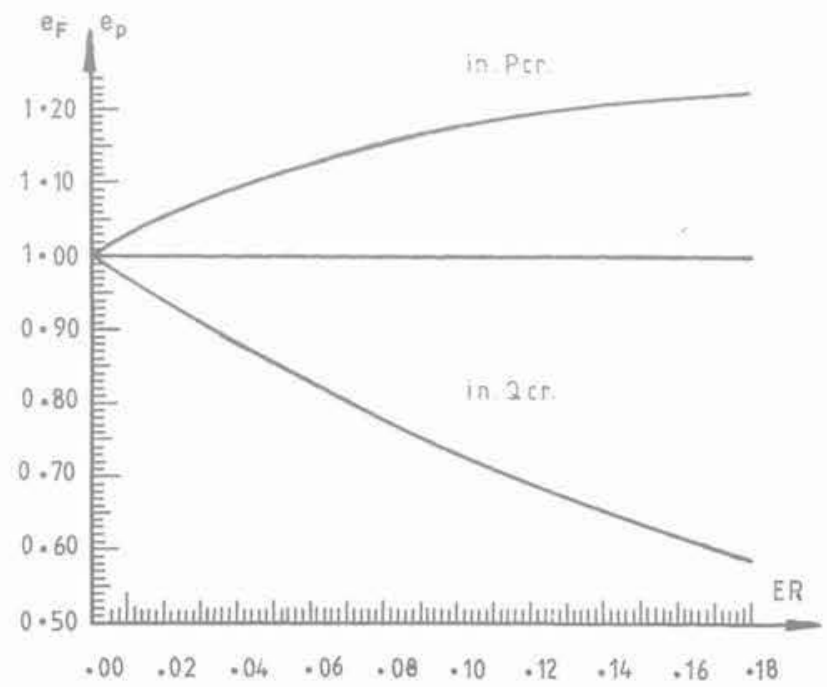

FIG. 6 Coefficient de correction de la force critique initiale et de la pression critique initiale en fonction de l'excentricité. où:

A est l'aire de la base de la fondation:

$\mathrm{c}_{\mathrm{a}}$ : l'adhésion entre le sol et la fondation;

$\mathrm{H} / \mathrm{V}$ : les composantes tangentielle et normale de la force sur la base de la fondation;

$\operatorname{tg} \delta$ : le coefficient de frottement entre la fondation et le sol.

Compte tenu des coefficients de correction présentés dans les graphiques de figures 6 et 7, en fonction de l'excentricité relative (er), de l'angle de frottement interne et de l'inclinaison relative $(\delta / \varnothing)$, l'expression de la pression critique initiale (4) peut être écrite sous la forme:

$$
\text { in. } p_{c r}=e_{p} i\left(\gamma \cdot b M_{\gamma}+q M_{q}+c M_{c}\right)
$$

où: $M_{\gamma} ; M_{q} ; M_{c}$ sont donnés par les relations $(3 \mathrm{~b})$ :

La force critique initiale, pour $\delta \neq 0$; er $\neq 0$, peut être calculée en fonction de la force critique correspondant à une charge verticale centrée $(\delta=0$; er $=0)$ en utilisant la relation:

$$
\text { in. } Q_{c r}(\delta \neq 0 \text { er } \neq 0)=e_{F} i \text { in. } Q_{c r}(\delta=0 \text {; er }=0)
$$

Les coefficients de corrélation $e_{p}$ et $e_{f}$ (Fig, 6), qui ne dépendent pas de la nature du sol de fondation, peuvent être utilisés aussi pour corriger les valeurs de la pression admissible $\left(\mathrm{q}_{\mathrm{ad}}\right)$, données dans des prescriptions techniques (en fonction de la nature du sol et des paramètres d'identification), vis-à-vis de l'excentricité de la charge:

$$
q_{a d}(e r \neq 0)=e_{p} q_{a d}(e r=0)
$$

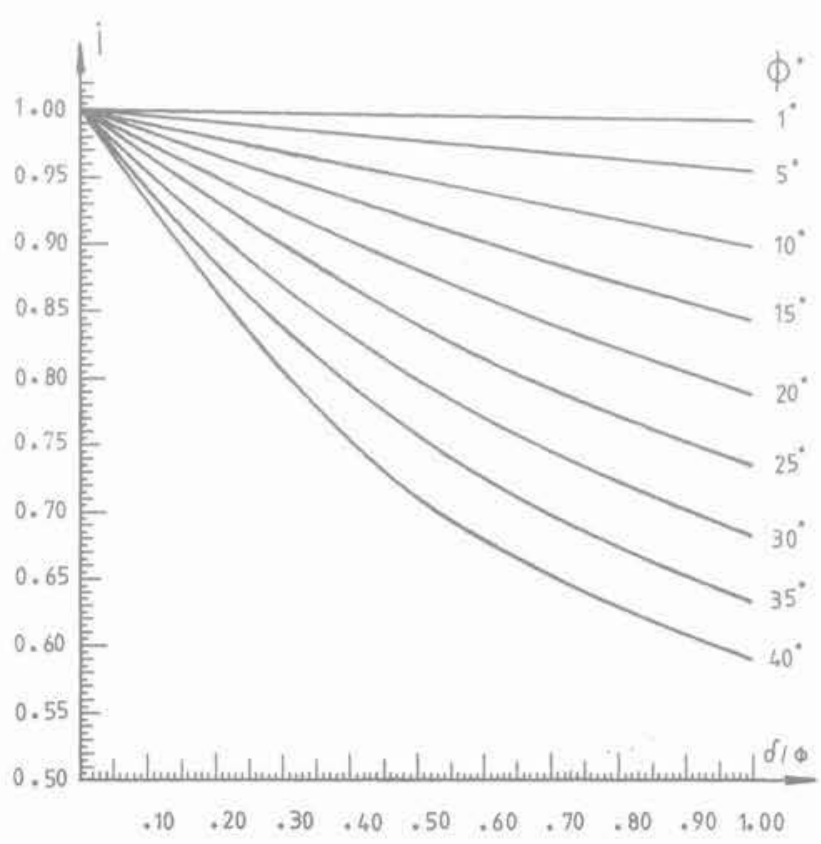

FIG.7 Facteur d'inclinaison en fonction de l'angle de frottement interne et de l'inclinaison relative. 


\section{Calcul approché de la pression critique initiale pour des fondations isolées}

Soit la semelle continue présentée à la figure 8 , et successivement soumise à la force critique initiale, puis à la force critique ultime. On suppose une répartition linéaire des pressions sur la base de la fondation pour la force critique initiale et une répartition uniforme (Meyerhof, 1953) pour la force critique ultime. Par conséquent les forces critiques sont reliées aux pressions critiques par les relations :

et

$$
\text { ult. } Q_{c r}=(b-2 e) \text { ult. } p_{c r} / \cos \delta
$$

$$
\text { in. } Q_{c r}=b \text { in. } p_{c r} /(1+6 e / b)
$$

où ult.Q est calculée en utilisant les relations de type Meyerhof, Hansen etc., et in.p $p_{c r}$ est calculée à l'aide de la relation 4.

Le rapport $F_{s}$ des forces critiques

$$
F_{s}=\text { ult } \cdot Q_{\mathrm{ce}} / \text { in. } Q_{\mathrm{cr}}
$$

s'écrit en utilisant les relations (19) :

$$
F_{s}=\frac{\text { ult. } p_{c r}}{\text { in. } p_{c r}} \frac{(1-2 \text { er })(1+6 \text { er })}{\cos \delta}
$$

Avec les notations:

$$
\begin{aligned}
& K=\frac{\text { ult. } p_{c r}(e r \neq 0 ; \delta \neq 0)}{\text { in. } p_{c r}(e r \neq 0 ; \delta \neq 0)} \\
& \frac{\text { in. } p_{c r}(e r=0 ; \delta=0)}{\text { ult. } p_{c r}(e r=0 ; \delta=0)}
\end{aligned}
$$

et

$$
\begin{aligned}
& F_{\text {so }}=\frac{\text { ult. } p_{c r}(e r=0 ; \delta=0)}{\text { in. } p_{c r}(e r=0 ; \delta=0)} \\
& F_{\text {sv }}=\frac{\text { ult. } p_{c r}(e r \neq 0 ; \delta \neq 0)}{\text { in. } p_{c r v}(e r \neq 0 ; \delta \neq 0)}
\end{aligned}
$$

les relations (20), donnant le rapport des forces critiques, deviennent respectivement:

$$
\mathrm{F}_{5}=K \mathrm{~F}_{\mathrm{s} 0}(1-2 \mathrm{er})(1+6 \mathrm{er}) / \cos \delta
$$

et

$$
F_{s}=F_{s y}(1-2 \text { er })(1+6 e r)
$$

où :

$$
F_{s \mathrm{v}}=\mathrm{KF}_{\mathrm{s0}} / \cos \delta
$$

Connaissant la valeur du rapport des pressions critiques verticales $\left(\mathrm{F}_{\mathrm{sv}}\right)$, donnée par la relation (22b), on calcule par la relation (22a) la pression critique initiale (composante verticale de la contrainte) en fonction de la pression critique ultime: (a)

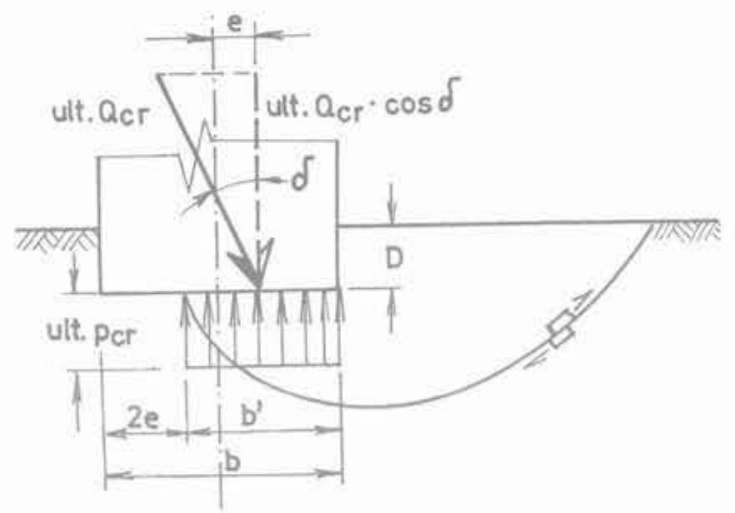

(b)

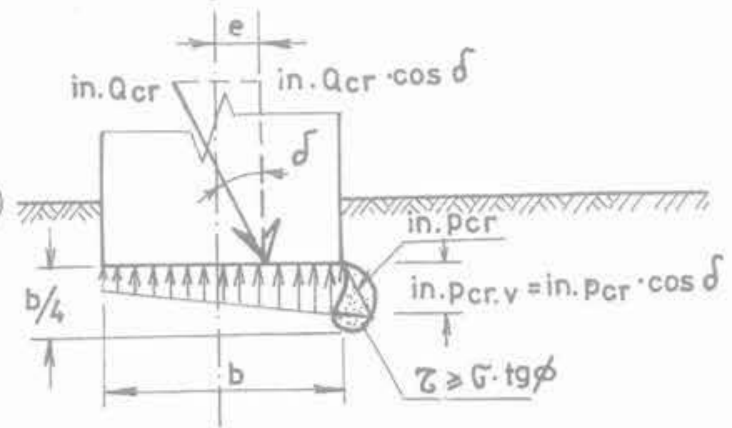

FIG, 8

Répartitions conventionnelles des pressions sous la base d'une fondation.

Les coefficients $\mathrm{K}$ et $\mathrm{F}_{\text {so' }}$ donnés par les équations (21) et qui définissent les rapports $F_{\mathrm{s}}$ et $\mathrm{F}_{\mathrm{sv}}$ par les relations (20c) et (22b), auront des formes spécifiques pour chaque méthode de calcul de la pression critique ultime.

Ainsi, pour une expression de la pression critique ayant la même structure que la formule de Meyerhof (Verdeyen et al., 1981):

$$
\begin{aligned}
\text { ult. } p_{c x}= & s_{c} d_{c} i_{c} c N_{c}+s_{q} d_{q} i_{q} \gamma d_{q} N_{q} \\
& +0,5 \gamma s_{\gamma} d_{\gamma} i_{\gamma} b^{\prime} N_{\gamma}
\end{aligned}
$$

et sachant que $\mathrm{s}_{\mathrm{c}}=\mathrm{s}_{\mathrm{q}}=\mathrm{s}_{\gamma}=1$, pour des semelles continues, on obtient:

où :

$$
\begin{gathered}
K=\left(a_{1}-2 \operatorname{er} i \gamma\right) /\left(e_{p} i s_{2}\right) \\
F_{s o}=d_{\gamma} a_{2} /\left(2 a_{3}\right)
\end{gathered}
$$

$$
\begin{aligned}
& a_{1}=i_{\gamma}+2(d / b) N_{q \gamma} i_{q}+2(c / \gamma b) N_{c r} d_{c \gamma} i_{c} \\
& a_{2}=1+2(d / b) N_{q \gamma}+2(c / \gamma b) N_{c \gamma} d_{c}(25 a) \\
& a_{3}=M_{\gamma} / N_{\gamma}+(d / b) N_{q} / N_{\gamma}+(c / \gamma b) M_{c} / N \gamma
\end{aligned}
$$

$e_{n} ; \mathrm{i}$ : coefficients de la pression critique initiale donnés par les expressions ( 3 ) pour er $=0$ et $\delta=0$;

$\mathrm{N}_{0 \gamma}=\mathrm{N} / \mathrm{N}_{\gamma}: \mathrm{N}_{c}=\mathrm{N} / \mathrm{N}_{\gamma}:$ rapports des coefficients de la pression critique utilme;

$\mathrm{d}_{\mathrm{cy}}=\mathrm{d}_{\mathrm{c}} / \mathrm{d}_{\gamma}$ : rapport des facteurs de profondeur;

$i_{y} ; i_{q} ; i_{c} ;$ facteurs d'inclinaison.

En revanche, pour une expression de la pression critique ultime ayant même structure que celle de la pression critique ultime présentée dans les normes soviétiques (SNIP 2.02.01-83):

$$
\text { ult. } p_{s t}=N_{\gamma} \zeta_{\gamma} b_{\gamma}^{\prime}+N_{q} \zeta_{q} \gamma_{d}+N_{c} \zeta_{q} c
$$

et sachant que $\zeta_{y}=\zeta_{\mathrm{q}}=\zeta_{\mathrm{c}}=1$ pour des semelles continues, on obtient: 


$$
\begin{gathered}
\mathrm{K}=\mathrm{N}_{\gamma}\left(\mathrm{a}_{1}-2 \mathrm{er}\right) /\left(\mathrm{a}_{2} \mathrm{~N}_{\gamma 0} \mathrm{e}_{\mathrm{p}} \mathrm{i}\right) \\
\mathrm{F}_{\mathrm{s} 0}=\mathrm{N}_{\gamma 0} \mathrm{a}_{2} /\left(\mathrm{M \gamma} \mathrm{a}_{3}\right)
\end{gathered}
$$

où :

$$
\begin{aligned}
& a_{1}=1+(d / b) N_{q \gamma}+(c / \gamma b) N_{c \gamma} \\
& a_{2}=1+(d / b) N_{q \gamma 0}+(c / \gamma b) N_{\gamma 0} \\
& a_{3}=1+(d / b) M_{q} / M_{\gamma}+(c / \gamma b) M_{c} / M_{\gamma}
\end{aligned}
$$

$e_{p} ; i ; N_{q} \gamma ; N_{c} \gamma ; M_{\gamma} ; M_{q} ; M_{c}$ tels que définis antérieurement.

$\mathrm{N}_{\mathrm{qro}}=\mathrm{N}_{\mathrm{qq}} / \mathrm{N}_{\mathrm{p}} ; \mathrm{N}_{\mathrm{cr} 0}=\mathrm{N} / \mathrm{co} / \mathrm{N}_{\mathrm{x}}$ : rapports des coefficients de la pression critique ultime pour une sollicitation verticale centrée (er $=0 ; \delta=0$ ).

Des expressions (24) et (25) il résulte que le rapport des pressions critiques normales $\mathrm{F}_{\mathrm{ci}}$ et le rapport des forces critiques normales $F_{\text {( }}>F_{\text {c }}$ ) peuvent être calculés en fonction de $\varnothing, \mathrm{c} / \gamma \mathrm{b}, \mathrm{d} / \mathrm{b}$, er et $\delta / \varnothing$, les résultats étant présentés dans des tables analogues à celles de l'annexe 4 a (où ult.p est calculée à l'aide de la formule de Meyerhof, 1963 et de Verdeyen et al., 1971) et de l'annexe $4 \mathrm{~b}$ (où ult.p $\mathrm{p}_{\text {ff }}$ est calculée à l'aide de la relation donnée dans la norme SNIP 2.02.01-83).

Si l'on admet l'hypothèse que le rapport des composantes normales des pressions critiques $\left(\mathrm{F}_{\mathrm{w}}\right)$ pour des fondations continues a pratiquement la même valeur pour des fondations isolées, alors la pression critique initiale et la force critique initiale (pour ce type de fondation) peuvent être estimées de façon approchée à partir de la relation (22a), soit par les expressions :

$$
\begin{aligned}
\text { in. } p_{\mathrm{crv}} & =\text { ult. } \mathrm{p}_{\mathrm{c}} / \mathrm{F}_{\mathrm{sv}} \\
\text { in. } \mathrm{Q}_{\mathrm{cr}} & =\text { ult. } \mathrm{Q}_{\mathrm{c}} / \mathrm{F}_{\mathrm{s}}
\end{aligned}
$$

Dans ces formules ult.p est calculée en employant les relations propres aux fondations isolées, $\mathrm{F}_{\mathrm{r} \text {. }}$ est déterminé à l'aide des tables de l'annexe 4 en fonction des paramètres $(\varphi, c / \gamma b, d / b, e r, \delta / \varphi)$ de la fondation étudiée en $F_{s}$ est obtenue à l'aide de l'abaque de la figure 9.

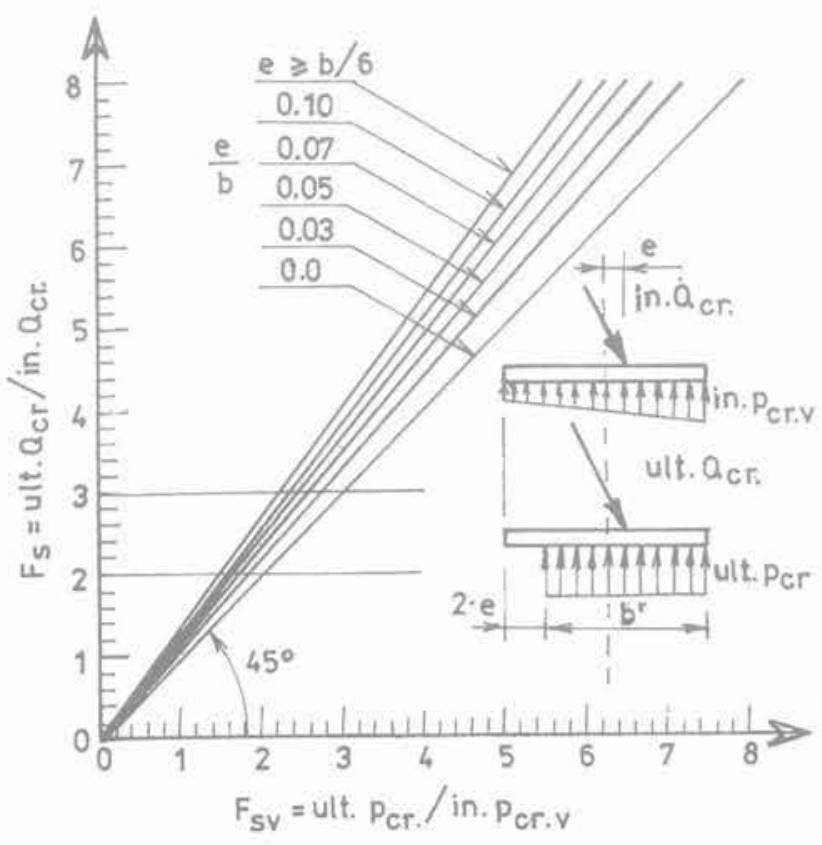

FIG: 9 Abaque du rapport des forces critiques en fonction du rapport des pressions critiques.
- Considérations sur le rapport des pressions critiques et sur la pression admissible

Si l'on modifie le graphique de la figure 1 en portant en abcisses, non pas la pression p, mais le rapport $\mathrm{S}=\mathrm{p} / \mathrm{in} . \mathrm{p}$, on obtient la courbe présentée à la figure 10 sur laquelle on peut déterminer approximativement la phase de compactage $\left(S_{f}=1\right)$ et la phase de cisaillement $\left(\mathrm{S}_{\mathrm{p}}=\mathrm{F}_{\mathrm{s}}-1\right)$.

En prenant pour pression admissible :

$$
q_{\text {add }}=\text { ult.p } p_{\mathrm{cr}} / \mathrm{F}_{\text {sacl }}
$$

et pour charge admissible :

$$
q_{\text {add }}=u l t \cdot Q_{c r} / F_{\text {s.ad }}
$$

où $F_{\text {sad }}$ est le coefficient de sécurité, on obtient en divisant par in.p :

$$
\begin{gathered}
q_{a d} / \text { in. } p_{c r}=\left(\text { ult. } p_{c t} / \text { in } p_{c r}\right) / F_{s} \text { ad } \\
q_{a d} / \text { in. } p f_{c r}=F_{s} / F_{s \text { sad }}
\end{gathered}
$$

et de même:

$$
q_{\mathrm{ad}} / \text { ult. } Q_{\mathrm{cr}}=\mathrm{F}_{\mathrm{s}} / \mathrm{F}_{\text {s.aid }}
$$

D'après les relations (29) et compte tenu que $F_{s}=S_{p}+1$ (Fig. 10), on peut considérer que pour:

$$
\mathrm{F}_{s} / \mathrm{F}_{s \mathrm{sd}}>1
$$$$
\mathrm{S}_{\mathrm{p}}>\mathrm{F}_{\mathrm{sad}}-1
$$

ou

le terrain de fondation a un comportement non linétaire et l'état de contraintes ne peut plus être calculé à partir des formules de Boussinesq.

La pression relative à la fin de la phase de cisaillement ( $\mathrm{S}_{p}$ ) (qui peut être déterminée aussi à partir des tables de l'annexe 4) est présentée à la figure 11 pour différentes méthodes de calcul de la pression critique ultime (Verdeyen et al., 1971; Bowles, 1983 ; SNIP 2.02.01-83) et dans le cas d'une charge verticale centrée.

On constate que la zone de cisaillement dépend principalement de l'angle de frottement interne et a des valeurs de $S_{p}$ comprises entre 0,01 et 3,8 à 9 suivant la

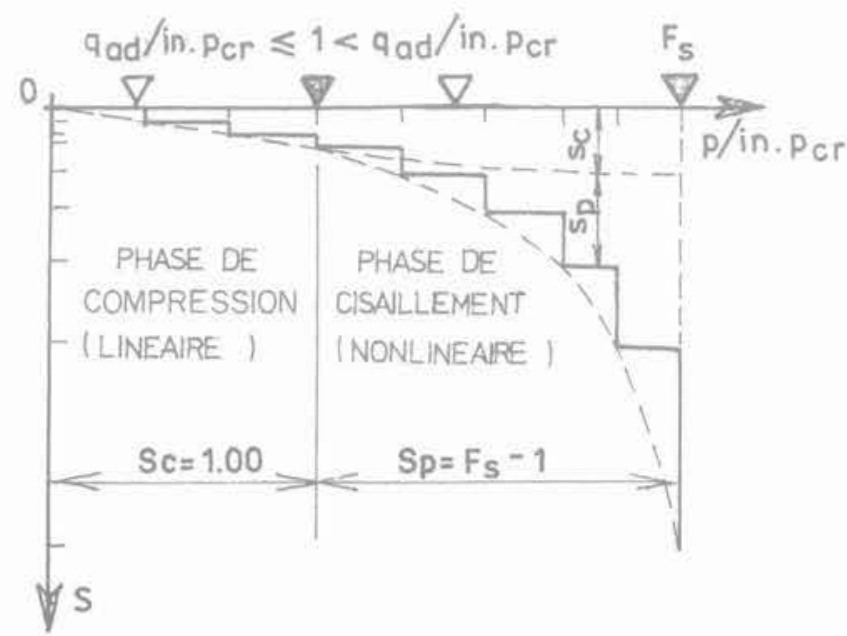

FG. 10 Pressions relatives correspondant aux phases de compression et de cisaillement du sol de fondation. 


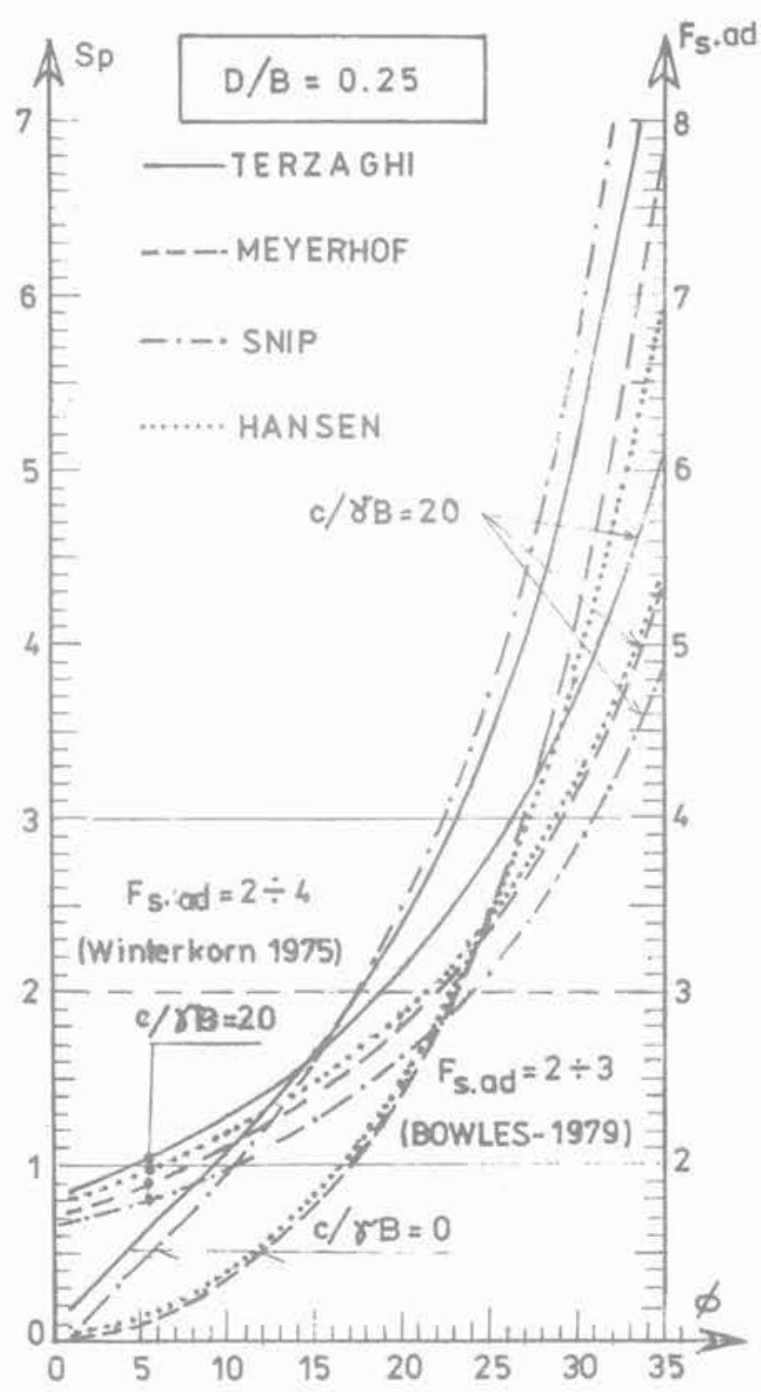

(a)

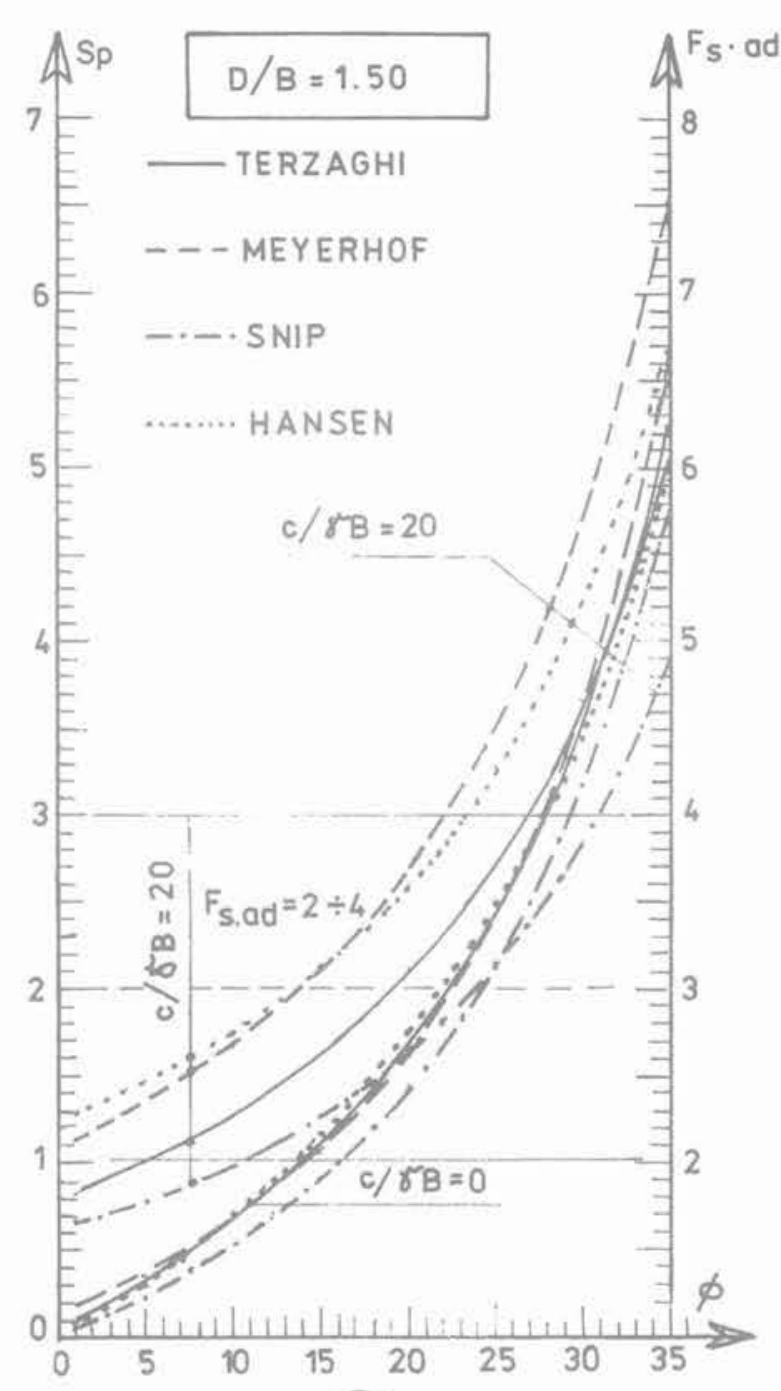

(b)

FQ.11 Variations de la pression relative enfin de phase de cisaillement en fonction de l'angle de frottement interne, de la cohésion et du coefficient de sécurité admissible.

méthode d'estimation de la pression critique ultime. La cohésion augmente en gếnéral la zone de cisaillement pour les sols à faible angle de frottement interne et la diminue aux grands angles de frottement interne (Fig. 11), de façon analogue à l'effet de la profondeur relative de la fondation (D/B).

Pour les valeurs usuelles du coefficient de sécurité, $F_{\text {s.ad }}=2$ à 3 (4) (Winterkorn, 1975; Bowles, 1979), le comportement linéaire du sol de fondation n'est assuré que dans le cas d'une phase de cisaillement relativement réduite: $S_{p}=1$ à 2 (3) (Fig. 11). Dans les autres cas, $\mathrm{S}_{\mathrm{p}}>2$ (3), la pression admissible s'accompagne d'un tassement plastique $\left(S_{p}\right)$ d'autant plus grand que la phase de cisaillement est plus importante.

Il convient donc soit d'adapter la valeur du coefficient de sécurité (Fig. 11) en fonction de la phase de cisaillement (ou de l'angle de frottement interne) soit de la méthode de calcul de ult. $\mathrm{p}_{\mathrm{cr}}$, pour que $\mathrm{p}_{\text {adm }}$ soit dans la phase du comportement linéaire, soit d utiliser un critère de tassement (dans le cas où $\mathrm{S}_{p}>2$ (3) incluant une composante plastique (voir relation 1a) dans le calcul du tassement total).

\section{Conclusions}

La détermination de la pression critique initiale d'une fondation superficielle permet d'établir le domaine de chargement pour lequel le sol a un comportement linéaire et de préciser la limite de validité de la formule de Boussinesq pour le calcul des contraintes et des tassements de consolidation primaire. En définissant et en estimant les pressions relatives correspondant à la zone de cisaillement, on peut soit adapter la valeur du coefficient de sécurité par rapport à la pression ou à la charge critique, soit imposer les valeurs maximales des tassements (consolidation primaire et plastique) correspondant à la pression admissible.

En utilisant des coefficients de corrélation établis $\left(e_{\text {, }}\right.$ i), on peut corriger les valeurs des pressions admissibles données dans les normes, pour tenir compte de chargements différents (centré/excentré/incliné). 
L'algorithme utilisé (paragraphe 2) pour le calcul de la pression critique initiale d'une semelle continue peut aussi être appliqué au cas de fondations isolées avec une expression (4) qui peut être évaluée sous la forme:

$$
\text { in. } p_{c r}=\gamma b M_{\gamma} s_{\gamma}+q M_{q} s_{q}+c M_{c} s_{c}
$$

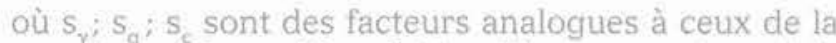
pression critique ultime. L'algorithme permet aussi de tenir compte de l'influence de la rigidité relative fondation-sol, sur la pression critique initiale, en utilisant des diagrammes localisés de pression à la base (Simvulidi, 1973). On peut également calculer la pression critique initiale pour différents degrés de consolidation du sol de fondation en introduisant les contraintes effec- tives dans l'expression de l'angle de déviation (10a). $\left(\sigma^{\prime}=\sigma-u\right)$ :

$$
\theta_{\max }^{\prime}=\arcsin \left[\frac{\sqrt{\left(\sigma_{z}-\sigma_{y}\right)^{2}+4\left(\tau_{z y}\right)^{2}}}{\left|\sigma_{z}+\sigma_{y}-2 u\right|+2 \cos ^{\prime} \operatorname{cotg} \varphi^{\prime}}\right]
$$

En vue d'une meilleure approche du comportement réel des terrains, on peut établir, en utilisant la méthode des éléments finis les coefficients de la pression critique $\left(\mathrm{M}_{\gamma} ; \mathrm{M}_{\mathrm{q}} ; \mathrm{M}_{\mathrm{c}}\right)$ et la valeur de la pression admissible correspondante pour des milieux à comportement élasto-plastique.

\section{Bibliographie}

Dorn S.W. McCracken D.D - Numerical Methods with Fortran. Chap. IV Case studies, John Wiley \& Sons, Inc. New York, 1970 .

Bowles E.J. - Physical and Geotechnica properties of Soils, Chap. 11 Consolidation and Settlements, McGraw Hill, Inc. 1979, pp. 299-320.

Foundation analysis and design, Chap. 4 Bearing capacity of foundations McGraw Hill International Book Com pany, Singapore, 1982, pp. 131-149

Costet J., Sanglerat G. - Cours pratique de mécanique des sols, Chap. 9 Fondations superficielles, Paris, Dunod, 1969, pp. 380-416.

Krol W. - Statique des fondations en béton armé compte tenu de la rigidité de la superstructure, Chap. 1 Comportement du sol, Paris, Dunod, 1971, pp. 29-41.
Masiov N.N. - Osnovî injenernoi geologiii mehaniki gruntov, Moskva, Vissaia skola, 1982, pp. 191-197.

Press W., et al. - Numerical Recipes, Cambridge University Press, 1986, p. 277.

Schlosser F. - Mécanique des sols, Paris, Presses de l'ENPC, 1983, pp. 31-32; 4748; 91-103.

SNIP 2.02.01-83 - Osnovania zganil i soorujenii. Stroitelinîe normî i pravilla SSSR, Moskva, 1985.

Simvulidi L.A. - Rascet injenernih kons truktii na uprugom osnovanii, Moskva, Vissaia skola, 1973.

Stanciu A. Boti N. - Precizari privind starea de tensiune din semiplanul elastic A VI-a Conferinta Nationala de Geotechnica Si Fundatii, Galati, România, 1987.
STAS 3300/2-85 - Terrain de fondation, Calcul du terrain sous les fondations superficielles.

Tomlinson J.M. - Foundation design and construction, Pitman Publishing, 1969

Tsytovich N, et al. - Foundation soils and substructures, Chap. 2 Fundamentals of foundation design, Moscow, Mir Publishers, 1974, pp. 48-56

Tsytovich N, - Soil Mechanics, Chap, 4 The theory of ultimate stresses of soils and its application, Moscow, Mir Publishers. 1976, pp. 123-148.

Verdeven J., et al. - Applications de la mécanique des sols (I), Chap. 5 Les fondations directes, Paris, Dunod, 1971, pp. $223-255$

Winterkorn F.H. et al. - Foundation engineering handbook, Chap. 3 Bearing capacity of shallow foundations, Van Nostrand Reinhold Company, 1975, pp. 143-145. 


\begin{tabular}{|c|c|c|c|c|c|c|c|c|c|c|c|c|c|c|c|c|c|c|c|c|c|c|c|c|c|c|}
\hline \multicolumn{3}{|c|}{$\Phi=1^{\circ}$} & \multicolumn{3}{|c|}{$\phi=5^{\circ}$} & \multicolumn{3}{|c|}{$\phi=10^{\circ}$} & \multicolumn{3}{|c|}{$\phi=15^{\circ}$} & \multicolumn{3}{|c|}{$\phi=20^{\circ}$} & \multicolumn{3}{|c|}{$\phi=25^{\circ}$} & \multicolumn{3}{|c|}{$\phi=30^{\circ}$} & \multicolumn{3}{|c|}{$\phi=35^{\circ}$} & \multicolumn{3}{|c|}{$\phi=40^{\circ}$} \\
\hline 1. & $\mathrm{Mq}$ & $M_{C}$ & $M_{s}$ & $\mathrm{Mq}$ & $M_{C}$ & $M_{*}$ & $\mathrm{Mq}$ & $M_{C}$ & $M_{4}$ & $\mathrm{Mg}$ & $M_{C}$ & $M_{*}$ & $\mathrm{Mg}$ & $M_{c}$ & $M_{7}$ & $\mathrm{Mq}_{\mathrm{q}}$ & $M_{C}$ & $M_{*}$ & $\mathrm{Mq}$ & $M_{c}$ & $M_{t}$ & $\mathrm{Mc}$ & $M_{C}$ & $M_{*}$ & $\mathrm{Mq}$ & $M_{C}$ \\
\hline
\end{tabular}

$$
\mathrm{e} / \mathrm{B}=0.0
$$

$(e=0.0 \cdot B / 6)$

\begin{tabular}{|c|}
\hline $0^{\circ}$ \\
\hline $1 / 4 \phi$ \\
\hline $1 / 2 \phi$ \\
\hline $3 / 4 \phi$ \\
\hline $1 / 1 \phi$ \\
\hline
\end{tabular}

\begin{tabular}{|r|l|l|l|l|l|l|l|l|l|l|l|l|l|l|l|l|l|l|l|l|l|l|l|l|l|l|l|}
\hline .01 & 1.06 & 3.23 & .08 & 1.32 & 3.61 & .18 & 1.73 & 4.17 & .32 & 2.30 & 4.84 & .51 & 3.06 & 5.66 & .78 & 4.11 & 6.67 & 1.15 & 5.59 & 7.95 & 1.68 & 7.71 & 9.58 & 2.46 & 10.85 & 11.73 \\
\hline .01 & 1.05 & 3.22 & .08 & 1.30 & 3.55 & .18 & 1.67 & 4.01 & .30 & 2.16 & 4.55 & .47 & 2.79 & 5.18 & .69 & 3.64 & 5.92 & .98 & 4.79 & 6.82 & 1.39 & 6.36 & 7.92 & 1.95 & 8.58 & 9.30 \\
\hline .01 & 1.05 & 3.21 & .08 & 1.27 & 3.49 & 17 & 1.60 & 3.87 & .29 & 2.02 & 4.29 & .43 & 2.56 & 4.77 & .62 & 3.26 & 5.33 & .86 & 4.18 & 5.99 & 1.19 & 5.43 & 6.78 & 1.63 & 7.15 & 7.76 \\
\hline .01 & 1.05 & 3.20 & .08 & 1.24 & 3.43 & .16 & 1.54 & 3.73 & .27 & 1.90 & 4.06 & .40 & 2.37 & 4.43 & .57 & 2.96 & 4.86 & .78 & 3.74 & 5.38 & 1.05 & 4.79 & 6.01 & 1.42 & 6.24 & 6.79 \\
\hline .01 & 1.04 & 3.19 & .07 & 1.22 & 3.37 & .16 & 1.48 & 3.61 & .26 & 1.80 & 3.86 & .38 & 2.21 & 4.15 & .53 & 2.73 & 4.51 & .71 & 3.43 & 4.94 & .96 & 4.37 & 5.48 & 1.30 & 5.66 & 6.18 \\
\hline
\end{tabular}

$$
e / B=0.04166667 \quad(e=0.25 \cdot B / 6)
$$

\begin{tabular}{|c|c|c|c|c|c|c|c|c|c|c|c|c|c|c|c|c|c|c|c|c|c|c|c|c|c|c|c|}
\hline $0^{\circ}$ & 02 & 15 & 56 & .09 & 4 & 97 & .20 & 89 & 57 & .36 & 51 & 30 & .56 & 34 & .19 & .85 & 4.49 & 7.30 & 1.26 & 6.11 & 8.70 & 1.84 & 8.44 & 10.50 & 2.70 & 11.00 & \\
\hline $4 \phi$ & 02 & 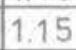 & 55 & .09 & 42 & 91 & 20 & 84 & 43 & 34 & 39 & 02 & .52 & 10 & & .77 & .06 & 6.59 & 1.10 & 38 & - & 156 & & & & .58 & \\
\hline $12 d$ & .02 & & & .08 & 40 & & 19 & & & 32 & & & & & & .70 & & & & & & 35 & & 7.73 & .87 & .21 & \\
\hline 149 & .02 & 1.15 & 53 & .08 & 1.38 & 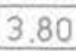 & 18 & 1.73 & 4.1 & 3 & 15 & & .4 & 2.69 & 5. & .65 & 3. & & .89 & 4. & 6.17 & 1.2 & - & 6.93 & 1.65 & 25 & 78 \\
\hline $11 \phi$ & .02 & 1.14 & 3.52 & .08 & 1.36 & 3.75 & 18 & 1.67 & 4.05 & 30 & 2.05 & 4.37 & 43 & 2.53 & 4.74 & 60 & 3.16 & 5.18 & .82 & 3.98 & 5.72 & 1.12 & 5.09 & 6.38 & 1.52 & 6.64 & 7.2 \\
\hline
\end{tabular}

\begin{tabular}{|c|c|c|c|c|c|c|c|c|c|c|c|c|c|c|c|c|c|c|c|c|c|c|c|c|c|c|c|}
\hline $0^{\circ}$ & .02 & 20 & 80 & 9 & 50 & 23 & 21 & 98 & 86 & .38 & 63 & 67 & .60 & 51 & 56 & 90 & 73 & 7.73 & 1.33 & 44 & 20 & 1.95 & 9 & 11 & 2.86 & & \\
\hline $4 \phi$ & .02 & 20 & 79 & & & & 21 & & 731 & .36 & 54 & & .56 & 31 & & 82 & 4.35 & 7.07 & 1.18 & & & 68 & 70 & & 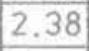 & & \\
\hline 29 & .02 & 20 & 78 & .09 & 48 & 4.12 & 20 & 90 & 60 & .34 & 43 & & .53 & 2 & 78 & .76 & 00 & 1 & 1.07 & & 7.39 & .48 & 79 & & .05 & & \\
\hline 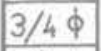 & 02 & 1.20 & 3.77 & .0 & 47 & $\theta$ & 20 & & 48 & .33 & 33 & & .5 & 4 & & .71 & 22 & & .98 & & & .34 & 6.14 & & 1.83 & & \\
\hline $1 \phi$ & 02 & 1.20 & 3.76 & .09 & 1.45 & 4.02 & 19 & 1.81 & 4.37 & .32 & 2.24 & 4.75 & .47 & 2.78 & 5.19 & 67 & 3.49 & 5.71 & .91 & 4.42 & 6.33 & 1.24 & 5.69 & 7.11 & 1.70 & 7.44 & 8,09 \\
\hline
\end{tabular}

$$
e / B=0.0833333 \quad(e=0.50 \cdot B / 6)
$$

\begin{tabular}{|c|c|c|c|c|c|c|c|c|c|c|c|c|c|c|c|c|c|c|c|c|c|c|c|c|c|c|c|}
\hline $0^{\circ}$ & .02 & 21 & .99 & 10 & 52 & 42 & .22 & 2.03 & .06 & .39 & 2.70 & 5.84 & .62 & .61 & .81 & .93 & 4.86 & .01 & 1.38 & 63 & .53 & .01 & 9.18 & 11.50 & 2.96 & & \\
\hline $4 \phi$ & .02 & 1.21 & 0 & 10 & 52 & 37 & .22 & 2.01 & 94 & .38 & 63 & 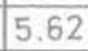 & .58 & 44 & - & .86 & & - & .24 & 2 & 59 & 76 & .08 & 06 & 2.50 & & \\
\hline $2 c$ & .02 & 1 & & 09 & & & 4 & 97 & & .5 & & & .55 & & & .80 & & & 13 & & & & .22 & 8.98 & 2.19 & & 0.42 \\
\hline 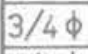 & .02 & 1.21 & .96 & .09 & 1.52 & & .21 & 1.94 & 4.71 & .35 & 2.46 & & & & & .75 & & & & & 7.25 & & & .21 & 1.98 & 8.69 & .42 \\
\hline 14 & .02 & 1.21 & 3.95 & .09 & 1.50 & 4.22 & .20 & 1.90 & 4.61 & .34 & 2.37 & 5.04 & .50 & 2.97 & 5.53 & 71 & 3.75 & 6.12 & 98 & 4.77 & 6.82 & 1.35 & 6.16 & .69 & 1.84 & 8.09 & 87 \\
\hline
\end{tabular}

$$
e / B=0.1250000 \quad(e=0.75 \cdot B / 6)
$$

\begin{tabular}{|c|c|c|c|c|c|c|c|c|c|c|c|c|c|c|c|c|c|c|c|c|c|c|c|c|c|c|c|}
\hline $0^{\circ}$ & .02 & 20 & 13 & 10 & 53 & 56 & 23 & 2.04 & 5.21 & .40 & .72 & 6.00 & 63 & 3.65 & .97 & 96 & .93 & 19 & & .73 & 9.74 & 0 & .33 & 75 & 02 & 0.18 & \\
\hline 4 & .02 & 20 & 4.12 & 10 & 53 & 5 & 22 & 2.03 & 5.09 & 39 & 2.67 & 5.79 & 60 & 3.52 & & 89 & & 63 & 28 & .19 & 8.86 & 1.82 & 8.32 & & 9 & 37 & 3 \\
\hline 2 & .02 & 1.20 & .11 & .10 & 53 & 6 & 22 & 2.01 & 4 & 37 & 2.61 & 59 & .57 & 3.38 & 30 & 83 & 38 & 4 & 18 & 5.71 & 8.15 & $1.64_{2}$ & 52 & & 29 & & \\
\hline 4 & .02 & 1.20 & 4.1 & 10 & 1.53 & & .22 & 1.99 & 4.8 & 3 & 2.54 & & .55 & 3.24 & & .7 & & & & & 7. & & 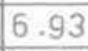 & & & & \\
\hline $1 \phi$ & .02 & 1.20 & 4.09 & .10 & 1.52 & 4.38 & .21 & 1.96 & 4.79 & .35 & 2.47 & 5.26 & .53 & 3.11 & 5.79 & .75 & 3.94 & 6.43 & 1.04 & 5.04 & 7.20 & 1.42 & 6.53 & 8.14 & 1.96 & 8.6 & 0.0 \\
\hline
\end{tabular}

$$
e / B=0.1666667 \quad(e=1.00 \cdot B / 6)
$$


Annexe $2 \mathrm{a}$ - Valeurs des forces critiques initiales pour les trois fondations $\left(\varphi=25^{\circ}\right)(\mathrm{KN})$

\begin{tabular}{|c|c|c|c|c|c|c|}
\hline er & $\delta$ & $0 \phi$ & $0.25 \phi$ & $0.50 \phi$ & $0.75 \phi$ & $1.0 \phi$ \\
\hline \multirow{3}{*}{0,0} & $F_{1}$ & 404.1515 & 360.6707 & 323.6819 & 294.7733 & 272.4534 \\
\cline { 2 - 7 } & $F_{2}$ & 1104.595 & 980.3044 & 880.2479 & 802.1035 & 741.7910 \\
\cline { 2 - 7 } & $F_{3}$ & 1959.368 & 1738.586 & 1580.601 & 1421.533 & 1314.175 \\
\hline \multirow{3}{*}{0416666} & $F_{1}$ & 355.7174 & 321.4052 & 292.5490 & 269.3596 & 251.1382 \\
\cline { 2 - 7 } & $F_{2}$ & 966.7891 & 873.4006 & 795.2285 & 732.5369 & 683.3376 \\
\cline { 2 - 7 } & $F_{3}$ & 1714.674 & 1549.190 & 1410.263 & 1298.704 & 1211.086 \\
\hline \multirow{3}{*}{0833333} & $F_{1}$ & 312.8665 & 286.8877 & 264.3217 & 245.7743 & 230.9967 \\
\cline { 2 - 7 } & $F_{2}$ & 850.8421 & 779.7126 & 718.3938 & 668.1829 & 628.2717 \\
\cline { 2 - 7 } & $F_{3}$ & 1508.463 & 1382.888 & 1274.121 & 1184.848 & 1113.781 \\
\hline \multirow{3}{*}{0.125} & $F_{1}$ & 276.9544 & 256.9612 & 239.1878 & 224.3341 & 212.3850 \\
\cline { 2 - 7 } & $F_{2}$ & 753.8494 & 698.6753 & 650.1528 & 609.8312 & 577.5155 \\
\cline { 2 - 7 } & $F_{3}$ & 1335.764 & 1238.831 & 1153.013 & 1081.446 & 1038.954 \\
\hline \multirow{3}{*}{0.16606} & $F_{1}$ & 2468322 & 231.1508 & 217.0075 & 205.0610 & 195.4040 \\
\cline { 2 - 7 } & $F_{2}$ & 672.6054 & 628.9142 & 590.059 & 557.4927 & 531.3048 \\
\cline { 2 - 7 } & $F_{3}$ & 1190.985 & 1114.676 & 1046.222 & 988.5717 & 942.0608 \\
\hline
\end{tabular}

Annexe $2 b$ - Valeurs des forces critiques initiales rapportées à la force centrée $(\delta=0 ; \mathrm{er}=0)$

\begin{tabular}{|c|c|c|c|c|c|c|}
\hline \multirow{3}{*}{0.9} & $F_{1}$ & $100 \%$ & 88.80200 & 79.69486 & 72.57718 & 67.08172 \\
\cline { 2 - 7 } & $F_{2}$ & $100 \%$ & 88.74781 & 79.68961 & 72.61512 & 67.15498 \\
\cline { 2 - 7 } & $F_{3}$ & $100 \%$ & 88.73195 & 80.66892 & 72.55059 & 67.07136 \\
\hline \multirow{3}{*}{0416666} & $F_{1}$ & 87.58243 & 79.13431 & 72.02951 & 66.31998 & 61.83362 \\
\cline { 2 - 7 } & $F_{2}$ & 87.52426 & 79.06971 & 71.99272 & 66.31720 & 61.86315 \\
\cline { 2 - 7 } & $F_{3}$ & 87.5116 & 79.06580 & 71.97539 & 66.28177 & 61.80999 \\
\hline \multirow{3}{*}{0833333} & $F_{1}$ & 77.03196 & 70.63564 & 65.07959 & 60.51296 & 56.87437 \\
\cline { 2 - 7 } & $F_{2}$ & 77.02747 & 70.58806 & 65.03681 & 60.49118 & 56.87798 \\
\cline { 2 - 7 } & $F_{3}$ & 76.98721 & 70.57826 & 65.02712 & 60.47092 & 56.84389 \\
\hline \multirow{3}{*}{0.125} & $F_{1}$ & 68.18992 & 63.26732 & 58.89127 & 55.23410 & 52.29206 \\
\cline { 2 - 7 } & $F_{2}$ & 68.24665 & 63.25168 & 58.85890 & 55.20855 & 52.28298 \\
\cline { 2 - 7 } & $F_{3}$ & 68.17320 & 63.22606 & 58.84616 & 55.19360 & 53.024 .95 \\
\hline \multirow{3}{*}{0.166666} & $F_{1}$ & 60.77344 & 56.91246 & 53.43020 & 50.48879 & 48.11110 \\
\cline { 2 - 7 } & $F_{2}$ & 60.89155 & 56.93615 & 53.41855 & 50.47029 & 48.09948 \\
\cline { 2 - 7 } & $F_{3}$ & 60.78411 & 56.88956 & 53.39592 & 50.45358 & 48.07981 \\
\hline
\end{tabular}

Annexe $2 c$ - Valeurs des forces critiques initiales rapportées aux forces verticales $(\delta=0$, er $\neq 0$ )

\begin{tabular}{|c|l|l|l|l|l|l|}
\hline \multirow{3}{*}{0.0} & $F_{1}$ & $100 \%$ & 88.80200 & 79.69486 & 72.57718 & 67.08172 \\
\cline { 2 - 7 } & $F_{2}$ & $100 \%$ & 88.74781 & 79.68961 & 72.61512 & 67.15498 \\
\cline { 2 - 7 } & $F_{3}$ & $100 \%$ & 88.73195 & 80.66892 & 72.55059 & 67.07136 \\
\hline \multirow{3}{*}{0416666} & $F_{1}$ & $100 \%$ & 90.35409 & 82.24197 & 75.72292 & 70.60048 \\
\cline { 2 - 7 } & $F_{2}$ & $100 \%$ & 90.34034 & 82.25459 & 75.77008 & 70.68115 \\
\cline { 2 - 7 } & $F_{3}$ & $100 \%$ & 90.34893 & 82.24668 & 75.74 .055 & 70.63062 \\
\hline \multirow{3}{*}{0833333} & $F_{1}$ & $100 \%$ & 91.69653 & 84.48388 & 78.55565 & 73.83218 \\
\cline { 2 - 7 } & $F_{2}$ & $100 \%$ & 91.64010 & 84.43327 & 78.53195 & 73.84116 \\
\cline { 2 - 8 } & $F_{3}$ & $100 \%$ & 91.67530 & 84.46484 & 78.54671 & 73.83549 \\
\hline \multirow{3}{*}{0.125} & $F_{1}$ & $100 \%$ & 92.78104 & 86.36359 & 81.00037 & 76.68591 \\
\cline { 2 - 7 } & $F_{2}$ & $100 \%$ & 92.68101 & 86.24438 & 80.89562 & 76.60887 \\
\cline { 2 - 8 } & $F_{3}$ & $100 \%$ & 92.74326 & 87.31860 & 80.96084 & 77.77975 \\
\hline \multirow{3}{*}{0.166666} & $F_{1}$ & $100 \%$ & 93.64692 & 87.91701 & 83.07706 & 79.16468 \\
\cline { 2 - 7 } & $F_{2}$ & $100 \%$ & 93.50419 & 87.72736 & 82.88554 & 78.99205 \\
\cline { 2 - 7 } & $F_{3}$ & $100 \%$ & 93.59281 & 87.84518 & 83.00455 & 78.09930 \\
\hline \hline MED & $100 \%$ & 91.41909 & 84.172987 & 78.162321 & 73.537318 \\
\hline
\end{tabular}


Annexe $3 a-$ Rapports des forces, pressions et coefficients pour $(\delta=0$, er $\neq 0)$

FORCE CRITIQUE INITIALE

\begin{tabular}{|c|c|c|c|c|c|}
\hline$\phi$ & 0 & .0416666 & .0833333 & .125 & .166666 \\
\hline $1^{\circ}$ & 1.0 & .87839 & .77409 & .68616 & .61223 \\
\hline $5^{\circ}$ & 1.0 & .87727 & .77287 & .68515 & .61154 \\
\hline $10^{\circ}$ & 1.0 & .87615 & .77144 & .68373 & .61023 \\
\hline $15^{\circ}$ & 1.0 & 87541 & .77042 & .68259 & .60904 \\
\hline $20^{\circ}$ & 1.0 & .87507 & .76989 & .68193 & .60825 \\
\hline $25^{\circ}$ & 1.0 & .87539 & .77016 & .68203 & .60816 \\
\hline $30^{\circ}$ & 1.0 & .87395 & .76907 & .68104 & .60714 \\
\hline $35^{\circ}$ & 1.0 & .87651 & .77174 & .68350 & .60921 \\
\hline $40^{\circ}$ & 1.0 & .87787 & .77360 & .68536 & .61081 \\
\hline MOY & 1.0 & .87623 & .77147 & .68350 & .60962 \\
\hline
\end{tabular}

PRESSION CRITIQUE INITIALE

\begin{tabular}{|c|l|l|l|l|}
\hline 0 & .0416666 & .083333 & .125 & 166666 \\
\hline 1.0 & 1.0980 & 1.1681 & 1.2008 & 1.2226 \\
\hline 1.0 & 1.0966 & 1.1593 & 1.1990 & 1.2231 \\
\hline 1.0 & 1.0952 & 1.1572 & 1.1987 & 1.2204 \\
\hline 1.0 & 1.0943 & 1.1556 & 1.1945 & 1.2181 \\
\hline 1.0 & 1.0938 & 1.1548 & 1.1934 & 1.2165 \\
\hline 1.0 & 1.0939 & 1.1549 & 1.1871 & 1.2130 \\
\hline 1.0 & 1.0945 & 1.1558 & 1.1941 & 1.2166 \\
\hline 1.0 & 1.0956 & 1.1576 & 1.1961 & 1.2184 \\
\hline 1.0 & 1.0973 & 1.1604 & 1.1994 & 1.2216 \\
\hline 1.0 & 1.0955 & 1.1574 & 1.1959 & 1.2193 \\
\hline
\end{tabular}

COEFFICIENTS $\mathrm{Mi}_{\mathrm{i}}$

\begin{tabular}{|c|c|c|c|c|}
\hline 0 & .041666 & 0833333 & 125 & 1666666 \\
\hline 1.0 & 1.0960 & 1.1534 & 1.1831 & 1.1925 \\
\hline 1.0 & 1.0968 & 1.1598 & 1.2001 & 1.2246 \\
\hline 1.0 & 1.0955 & 1.1582 & 1.1988 & 1.2242 \\
\hline 1.0 & 1.0945 & 1.1567 & 1.1968 & 1.2218 \\
\hline 1.0 & 1.0941 & 1.1558 & 1.1954 & 1.2199 \\
\hline 1.0 & 1.0941 & 1.1557 & 1.1950 & 1.2190 \\
\hline 1.0 & 1.0947 & 1.1565 & 1.1956 & 1.2191 \\
\hline 1.0 & 1.0958 & 1.1582 & 1.1974 & 1.2206 \\
\hline 1.0 & 1.0975 & 1.1609 & 1.2004 & 1.2234 \\
\hline 1.0 & 1.0954 & 1.1573 & 1.1958 & 1.2184 \\
\hline
\end{tabular}

Annexe $3 \mathrm{~b}$ - Rapports moyens des forces, pressions et coefficients en fonction de $\delta$ (er $\neq 0$ )

FORCE CRITIQUE INITALE

\begin{tabular}{|c|c|c|c|c|c|}
\hline$\phi \delta$ & $0^{\circ}$ & $0.25 \phi$ & $0.50 \phi$ & $0.75 \phi$ & $1.0 \phi$ \\
\hline $1^{\circ}$ & 1.0 & .99817 & .99632 & .99444 & .99254 \\
\hline $5^{\circ}$ & 1.0 & .98924 & .97811 & .96674 & .95524 \\
\hline $10^{\circ}$ & 1.0 & .97577 & .94953 & .92456 & .90048 \\
\hline $15^{\circ}$ & 1.0 & .95749 & .91640 & .87824 & .84364 \\
\hline $20^{\circ}$ & 1.0 & .93717 & .88089 & 83015 & .78793 \\
\hline $25^{\circ}$ & 1.0 & .91419 & .84173 & .78162 & .73573 \\
\hline $30^{\circ}$ & 1.0 & .88790 & .79978 & .73293 & .68373 \\
\hline $35^{\circ}$ & 1.0 & .86006 & .75751 & .68527 & .63587 \\
\hline $40^{\circ}$ & 1.0 & .83169 & .71354 & .63793 & .59007 \\
\hline
\end{tabular}

PRESSION CRITIQUE INITIALE

\begin{tabular}{|l|l|l|l|l|}
\hline $0^{\circ}$ & $0.25 \phi$ & $0.50 \phi$ & $0.75 \phi$ & $1.0 \phi$ \\
\hline 1.0 & .99817 & .99633 & .99444 & .99254 \\
\hline 1.0 & .98924 & .97811 & .96676 & .95522 \\
\hline 1.0 & .97455 & .94918 & .924 .22 & .90015 \\
\hline 1.0 & .95747 & .91640 & .87824 & .84364 \\
\hline 1.0 & .93762 & .87996 & .83016 & .78792 \\
\hline 1.0 & .91561 & .84200 & .77901 & .73546 \\
\hline 1.0 & .88843 & .80007 & .73358 & .68391 \\
\hline 1.0 & .86006 & .75751 & .68527 & .63587 \\
\hline 1.0 & .82895 & .71320 & .63793 & .59007 \\
\hline
\end{tabular}

COEFFICIENTS $\mathrm{Mi}_{\mathrm{i}}$

\begin{tabular}{|c|c|c|c|c|}
\hline $0^{\circ}$ & $0.25 \phi$ & $0.50 \phi$ & $0.75 \phi$ & $1.0 \phi$ \\
\hline 1.0 & .99871 & .99735 & .99595 & .99448 \\
\hline 1.0 & .98925 & .97812 & .96680 & .95528 \\
\hline 1.0 & .97460 & .94908 & .92409 & .90006 \\
\hline 1.0 & .95701 & .91583 & .87872 & .84312 \\
\hline 1.0 & .93817 & .87941 & .82977 & .78776 \\
\hline 1.0 & .91366 & .84055 & .78131 & .73499 \\
\hline 1.0 & .88824 & .79970 & .73302 & .68399 \\
\hline 1.0 & .85969 & .75722 & .68517 & .63595 \\
\hline 1.0 & .82865 & .71333 & .63787 & .59012 \\
\hline
\end{tabular}


Annexe $4 \mathrm{a}$ - Rapport des composantes normales des pressions critiques établies $\left(\mathrm{F}_{\mathrm{sv}}\right)$ selon la formule de Meyerhof (1963)

\begin{tabular}{|c|c|c|c|c|c|c|c|c|c|c|c|c|c|c|c|c|c|c|c|c|c|c|c|c|c|c|}
\hline \multirow{3}{*}{$\frac{\mathrm{e}}{\mathrm{B}}$} & \multirow{3}{*}{\multicolumn{2}{|c|}{$\frac{D}{B} \frac{c}{\gamma^{\prime} B}$}} & \multicolumn{3}{|c|}{$1^{\circ}$} & \multicolumn{3}{|c|}{$5^{\circ}$} & \multicolumn{3}{|c|}{$10^{\circ}$} & \multicolumn{3}{|c|}{$15^{\circ}$} & \multicolumn{3}{|c|}{$20^{\circ}$} & \multicolumn{3}{|c|}{$25^{\circ}$} & \multicolumn{3}{|c|}{$30^{\circ}$} & \multicolumn{3}{|c|}{$35^{\circ}$} \\
\hline & & & \multicolumn{3}{|c|}{$\delta / \phi$} & \multicolumn{3}{|c|}{$\delta / \varnothing$} & \multicolumn{3}{|c|}{$\delta / \phi$} & \multicolumn{3}{|c|}{$\delta / \phi$} & \multicolumn{3}{|c|}{$\delta / \varnothing$} & \multicolumn{3}{|c|}{$\delta / \phi$} & \multicolumn{3}{|c|}{$\delta / \phi$} & \multicolumn{3}{|c|}{$\delta / \phi$} \\
\hline & & & 0 & .5 & 1 & 0 & .5 & 1 & 0 & .5 & 1 & 0 & .5 & 1 & 0 & .5 & 1 & 0 & .5 & 1 & 0 & .5 & 1 & 0 & .5 & 1 \\
\hline
\end{tabular}

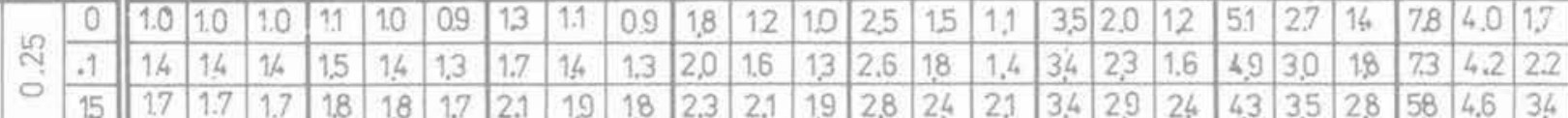

\begin{tabular}{l|l|l|l|l|l|l|l|l|l|l|l|l|l|l|l|l|l|l|l|l|l|l|l|l|l|}
\hline & 0 & 1.1 & 1.1 & 1.1 & 1.2 & 1,2 & 1.1 & 1.5 & 1,3 & 1.2 & 1.9 & 1.5 & 1.4 & 2.5 & 1.9 & 1.5 & 3.3 & 2.4 & 18 & 4.6 & 3.2 & 2.2 & 6.7 & 4.4 & 2.7 \\
\hline
\end{tabular}

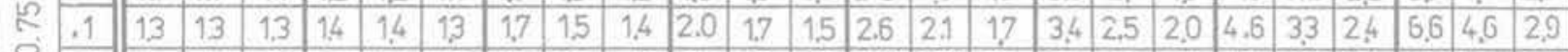

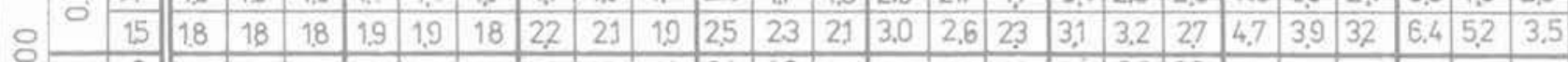

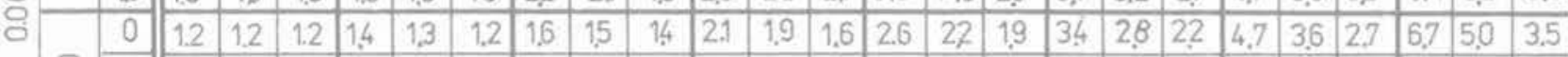

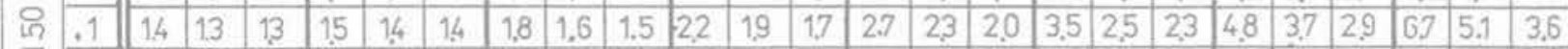

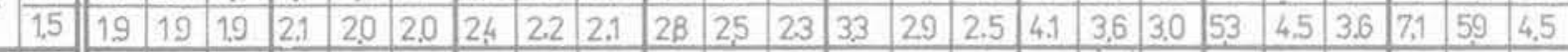

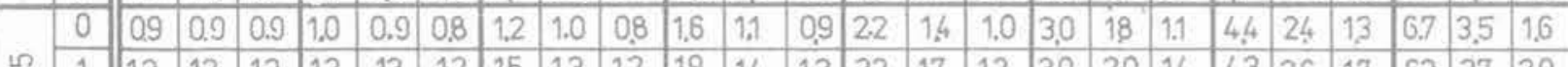

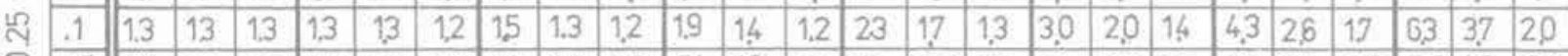

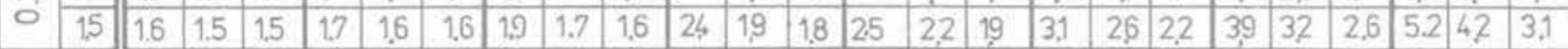

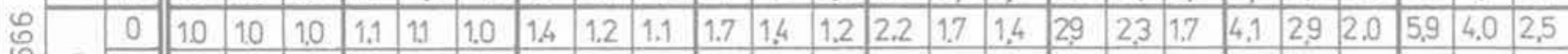

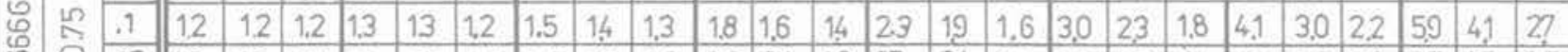

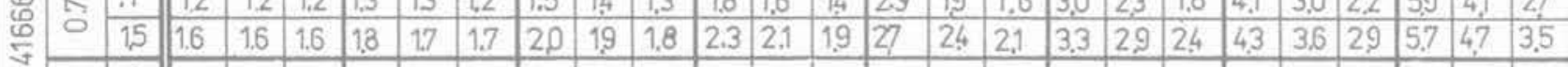

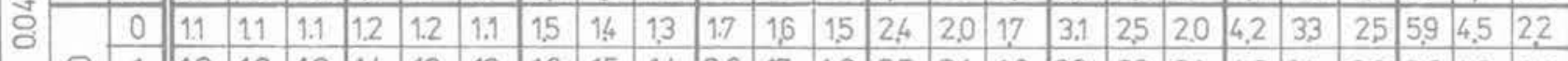

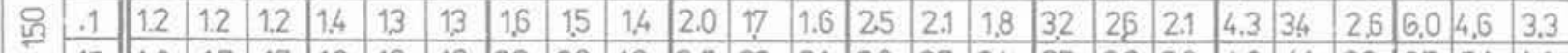

\begin{tabular}{|l|lllllllllllllllllllllllll|l|l|l|l|}
15 & 1.8 & 1.7 & 1.7 & 1.9 & 1.9 & 19 & 22 & 2.0 & 1.9 & 2.5 & 23 & 2.1 & 3.0 & 27 & 2.4 & 3.7 & 3.2 & 2.8 & 4.8 & 4.1 & 3.3 & 6.5 & 5.4 & 4.1 \\
\hline
\end{tabular}

\begin{tabular}{ll|lll|llllllllllll|l|l|l|l|l|l|l|l|l|l|l|}
\hline & 0 & 0.9 & 0.9 & 0.9 & 0.9 & 0.8 & 0.8 & 1.1 & 0.9 & 0.8 & 1.4 & 1.0 & 0.8 & 2.0 & 1.3 & 0.9 & 2.7 & 1.6 & 1.0 & 3.9 & 22 & 12 & 6.0 & 32 & 1.5 \\
\hline
\end{tabular}

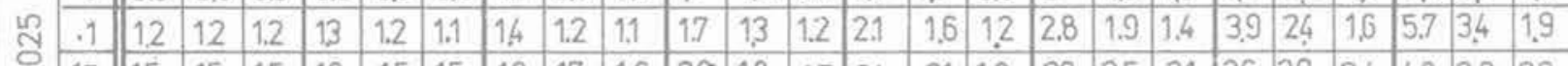

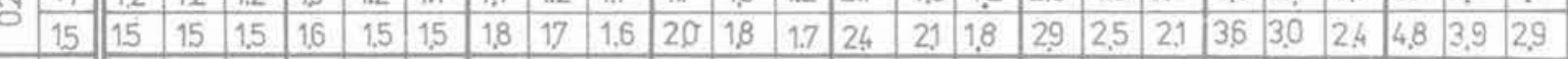

\begin{tabular}{|l|l|l|l|l|l|l|l|l|l|l|l|l|l|l|l|l|l|l|l|l|l|l|l|l|}
\hline & 0.9 & 0.9 & 0.9 & 1.1 & 1.0 & 1.0 & 1.3 & 1.1 & 1.0 & 1.6 & 1,3 & 1.2 & 2.0 & 1.6 & 1.3 & 2.7 & 2.0 & 1.6 & 3.7 & 2.7 & 1.9 & 5.4 & 3.7 & 2.4 \\
\hline
\end{tabular}

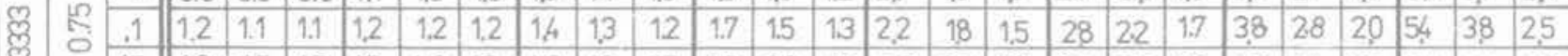

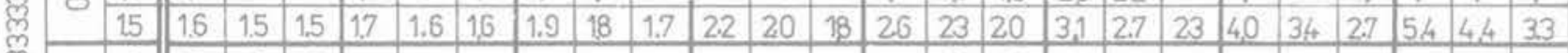

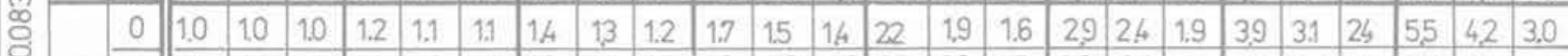

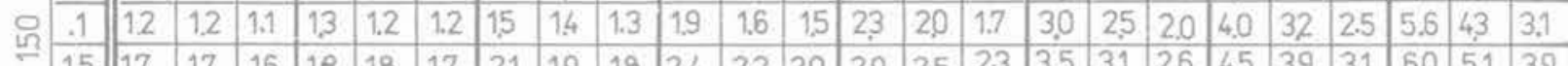

\begin{tabular}{|l|lllllllllllllllllllllllllllllll|}
1.5 & 1.5 & 1.7 & 1.6 & 18 & 1.8 & 1.7 & 2.1 & 1.9 & 1.8 & 2.4 & 2.2 & 20 & 2.9 & 2.5 & 2.3 & 3.5 & 3.1 & 2.6 & 4.5 & 3.9 & 3.1 & 6.0 & 5.1 & 3.9 \\
\hline
\end{tabular}

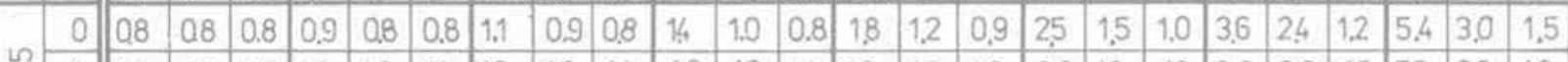

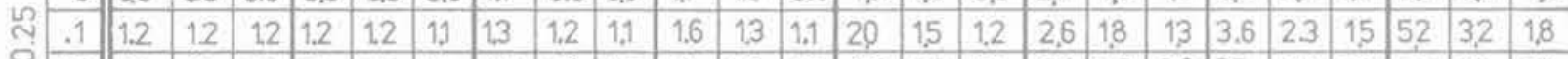

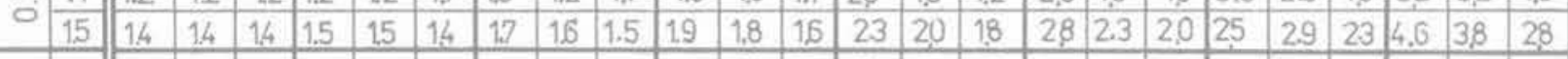

\begin{tabular}{|l|lllllllll|l|l|l|l|l|l|l|l|l|l|l|l|l|l|l|l|}
\hline \multirow{2}{*}{$\mathrm{n}$} & 0 & 0.9 & 0.9 & $\mathrm{ag}$ & 1.0 & 1.0 & 0.9 & 12 & 1.1 & 1.0 & 1.5 & 1.3 & 1.1 & 1.9 & 15.5 & 1.3 & 2.6 & 1.9 & 1.5 & 3.5 & 2.5 & 1.8 & 5.0 & 3.5 & 2.3 \\
\hline
\end{tabular}

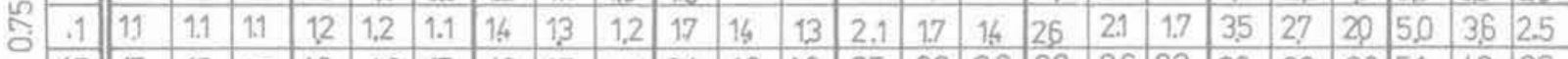

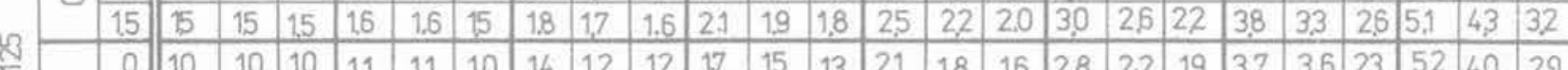

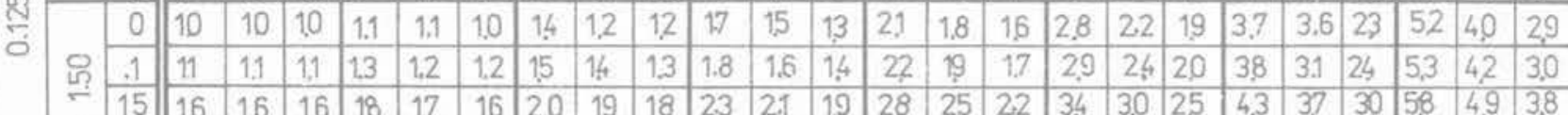

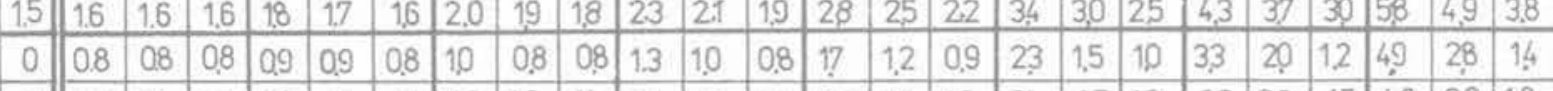

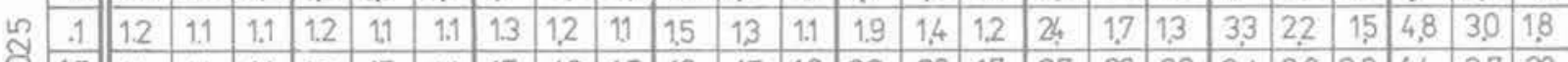

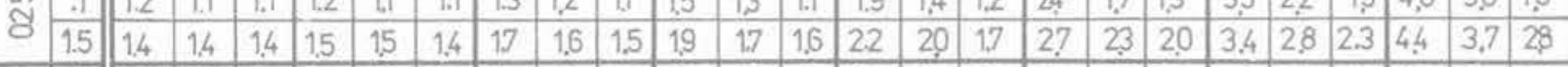

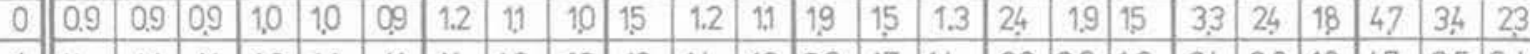

\begin{tabular}{|c|c|c|c|c|c|c|c|c|c|c|c|c|c|c|c|c|c|c|c|c|c|c|c|c|c|}
\hline 0 & 11 & 1.1 & 1.1 & 1.2 & 1.1 & 1.1 & 1.4 & 1.2 & 12 & 16 & 1.4 & 1.3 & 2.0 & 1.7 & 1.4 & 2.6 & 2.0 & 1.6 & 3.4 & 2.6 & 1.9 & 4.7 & 3.5 & 2.4 \\
\hline
\end{tabular}

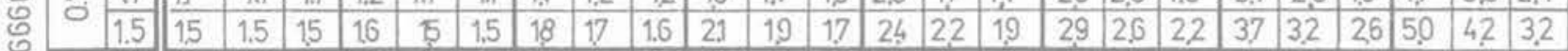

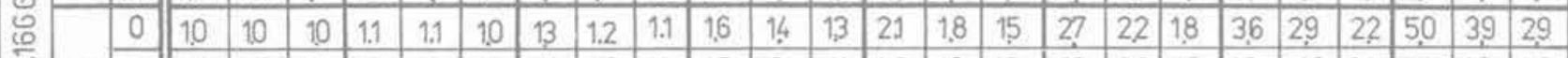

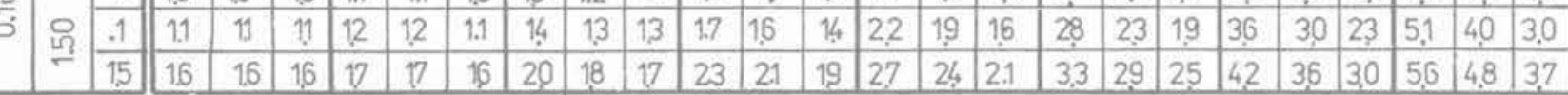


Annexe $4 b$ - Rapport des composantes normales des pressions critiques établies $\left(\mathrm{F}_{\mathrm{sv}}\right)$ selon la formule du SNIP 2.02.01.83

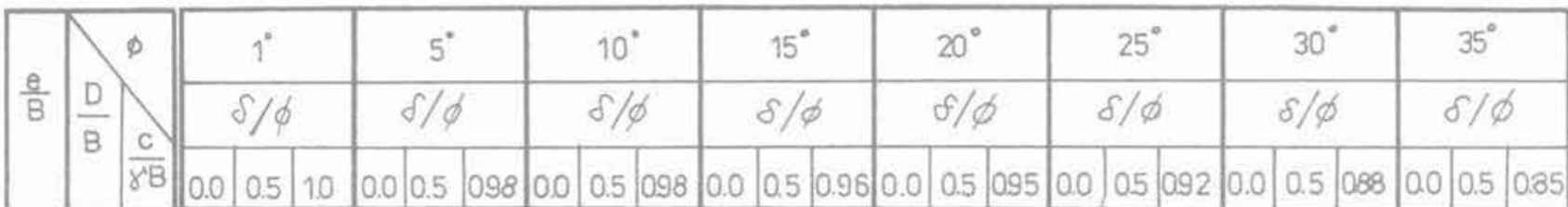

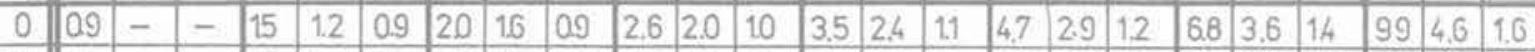

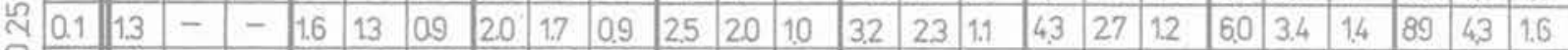

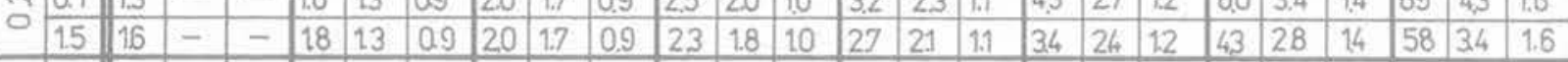

\begin{tabular}{|l|l|l|l|l|l|l|l|l|l|l|l|l|l|l|l|l|l|l|l|l|l|l|l|l|l|}
\hline & 0 & 0.9 & - & - & 1.3 & 1.1 & 1.0 & 1.7 & 1.5 & 1.0 & 2.1 & 1.7 & 1.0 & 27 & 2.0 & 1.1 & 3.6 & 24 & 12 & 4.9 & 2.9 & 1.4 & 70 & 37 & 1.6 \\
\hline
\end{tabular}

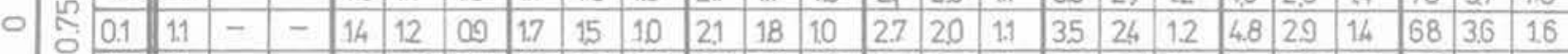

\begin{tabular}{|l|l|l|l|l|l|l|l|l|l|l|l|l|l|l|l|l|l|l|l|l|l|l|l|l|}
\hline 1.5 & 15 & - & - & 1.7 & 1.3 & 0.9 & 20 & 1.6 & 0.9 & 22 & 1.8 & 1.0 & 27 & 2.0 & 11 & 32 & 23 & 1.2 & 4.1 & 2.7 & 1.3 & 56 & 3.3 & 1.6 \\
\hline
\end{tabular}

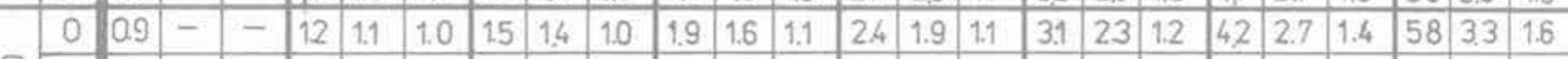

\begin{tabular}{|l|l|l|l|l|l|l|l|l|l|l|l|l|l|l|l|l|l|l|l|l|l|l|l|l|}
\hline \multirow{2}{*}{ a. } & 10 & - & - & 1.3 & 1.1 & 1.0 & 1.6 & 1.4 & 1.0 & 2.0 & 1.7 & 1.1 & 2.5 & 1.9 & 1.1 & 3.1 & 2.3 & 1.2 & 4.2 & 2.7 & 1.4 & 58 & 3.3 & 1.6 \\
\hline
\end{tabular}

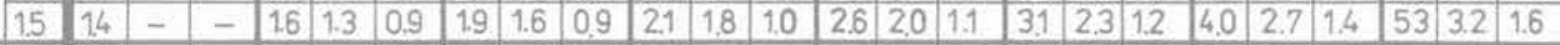

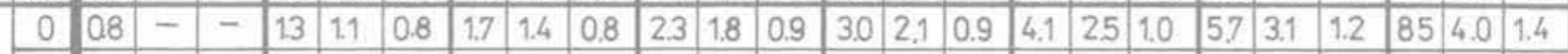

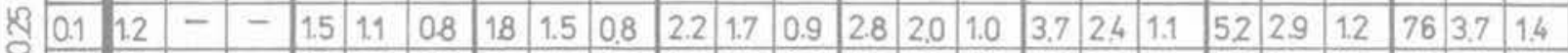

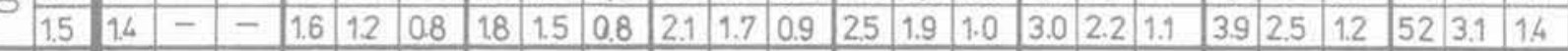

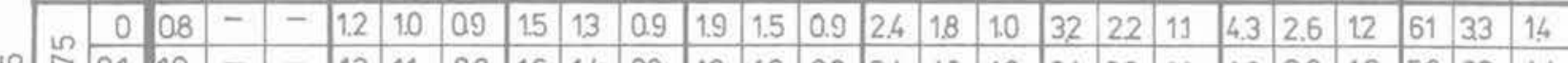

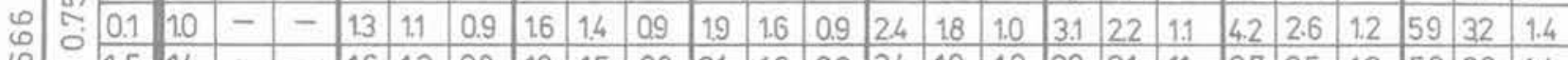

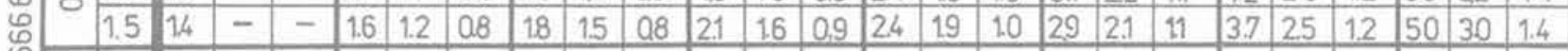

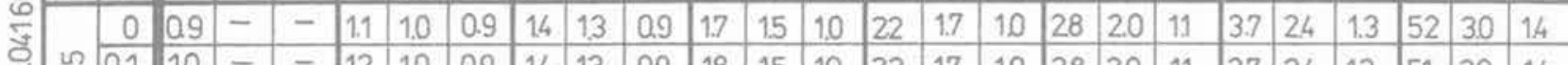

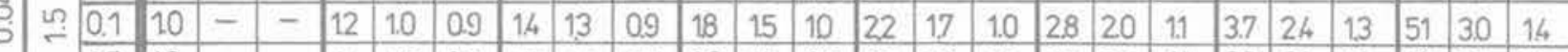

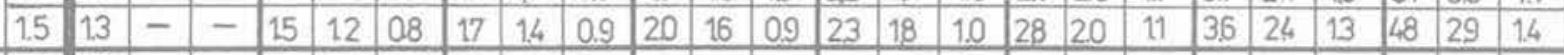

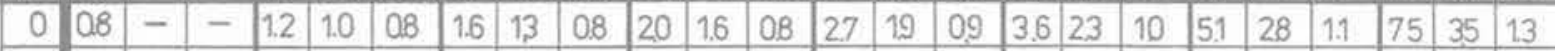

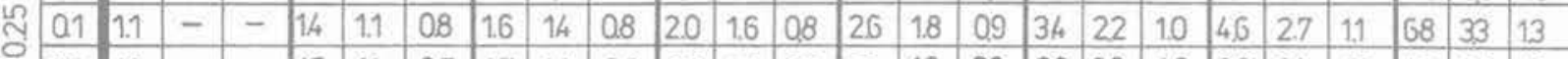

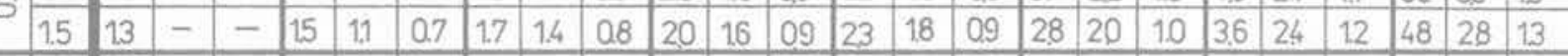

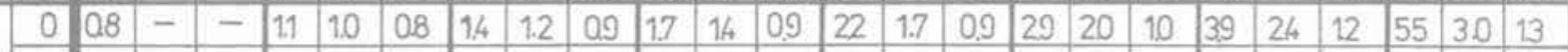

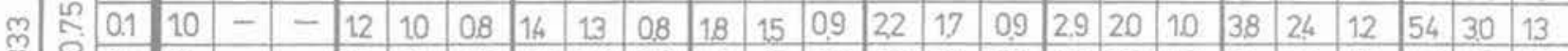

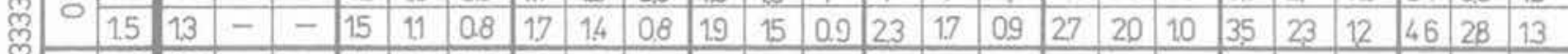

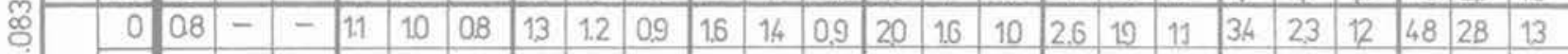

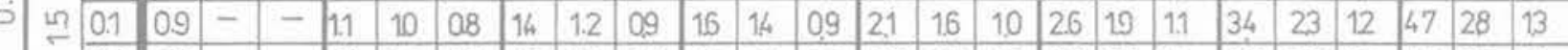

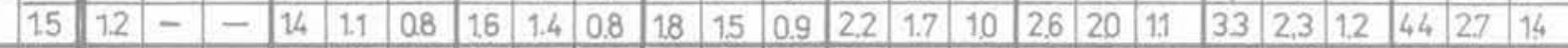

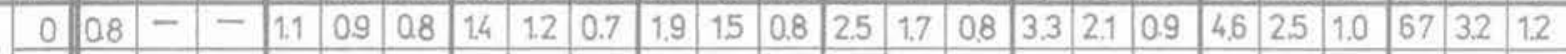

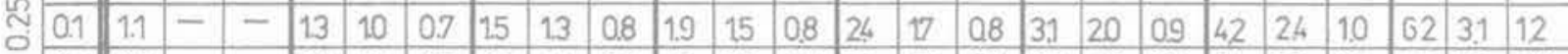

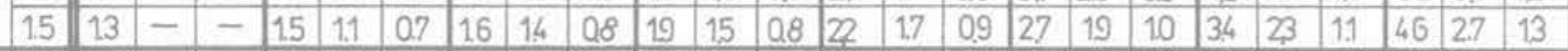

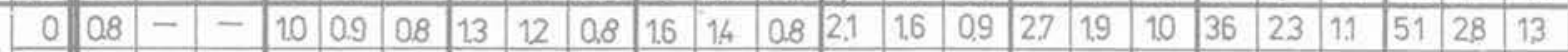

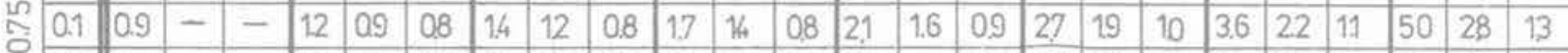

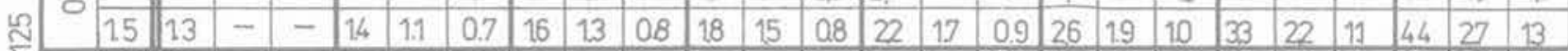

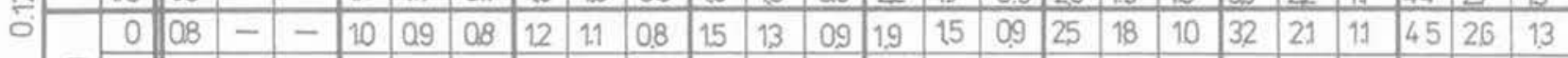

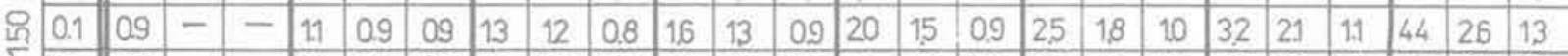

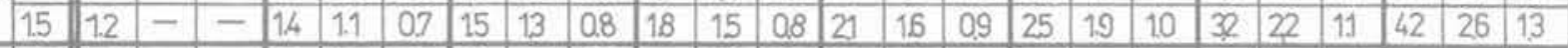

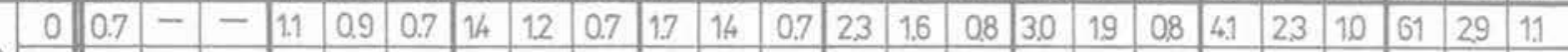

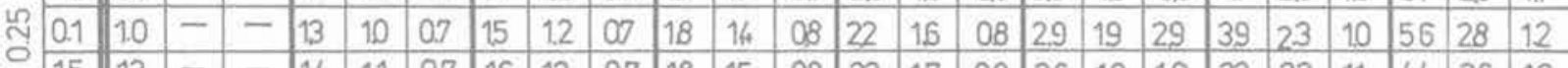

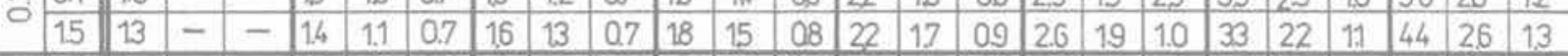

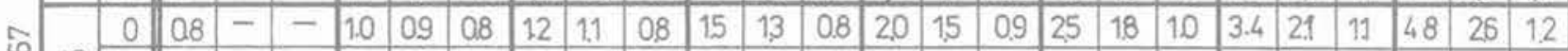

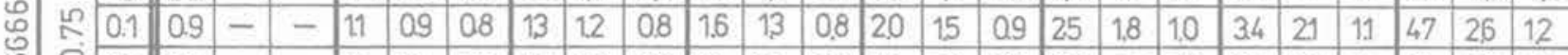

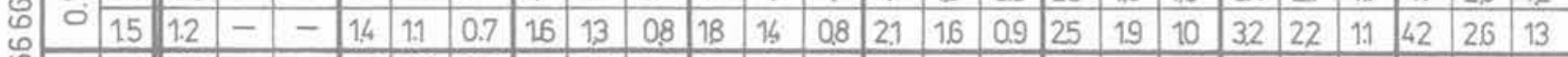

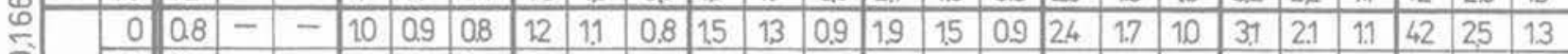

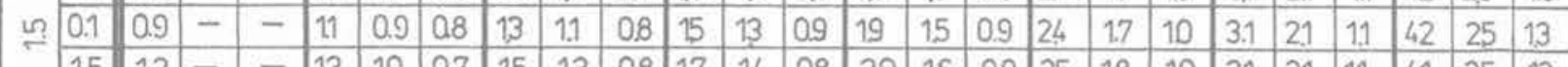

